SLAC-PUB-9257

October 2002

\title{
Cosmology and the Standard Model
}

\author{
James D. Bjorken \\ Stanford Linear Accelerator Center \\ Stanford University, Stanford, California 94309 \\ E-mail: bjorken@slac.stanford.edu
}

\begin{abstract}
We consider the properties of an ensemble of universes as function of size, where size is defined in terms of the asymptotic value of the Hubble constant (or, equivalently, the value of the cosmological constant). We assume that standard model parameters depend upon size in a manner that we have previously suggested, and provide additional motivation for that choice. Given these assumptions, it follows that universes with different sizes will have different physical properties, and we estimate, very roughly, that only if a universe has a size within a factor $\sqrt{2}$ of our own will it support life as we know it. We discuss implications of this picture for some of the basic problems of cosmology and particle physics, as well as the difficulties this point of view creates.
\end{abstract}

\footnotetext{
*Work supported by Department of Energy contract DE-AC03-76SF00515.
} 


\section{Introduction}

Our universe seems, according to the present-day evidence, to be spatially flat and to possess a nonvanishing cosmological constant [1]. These features, while not yet rocksolid experimentally, are hardly what would have been anticipated by the founding fathers of cosmology. The cosmological constant in particular is, for cosmologists and general relativists, the Great Mistake. And for elementary particle physicists it is the Great Embarrassment. It is fair to say that each school would just as soon see it go away. But in this paper we assume that it will not do so, and that the present evidence will prevail.

The cosmological constant is a peculiar quantity. By definition it has something to do with cosmology. But it also has something to do with the local structure of elementary particle physics, where it represents the stress-energy density $\mu$ of the vacuum.

$$
\mathrm{E}_{c c}=\int d^{4} x \sqrt{-g} \mu^{4}=\frac{1}{8 \pi G} \int d^{4} x \sqrt{-g} \Lambda .
$$

Instead of the parameter $\mu$, the cosmologist will use $\Lambda$, as defined above, but expressed in terms of the properties of the spacetime of our distant future, a future dominated by dark energy and exponential expansion:

$$
d s^{2}=d \hat{t}^{2}-e^{2 H_{\infty} \hat{t}}\left(d \hat{r}^{2}+\hat{r}^{2} d \theta^{2}+\hat{r}^{2} \sin ^{2} \theta d \phi^{2}\right) .
$$

This matter-free spacetime is equivalent to static DeSitter space, characterized by a horizon radius $R_{\infty}$,

$$
d s^{2}=\left(1-\frac{r^{2}}{R_{\infty}^{2}}\right) d t^{2}-\left(1-\frac{r^{2}}{R_{\infty}^{2}}\right)^{-1} d r^{2}-r^{2}\left(d \theta^{2}+\sin ^{2} \theta d \phi^{2}\right)
$$

via the coordinate transformations

$$
\begin{aligned}
r & =\hat{r} e^{H_{\infty} \hat{t}} \\
t & =\hat{t}-\frac{1}{2 H_{\infty}} \ln \left(1-H_{\infty}^{2} r^{2}\right) .
\end{aligned}
$$


This horizon radius is determined in terms of the asymptotic Hubble constant

$$
\begin{aligned}
& H_{\infty}=\lim _{t \rightarrow \infty} H(t) \\
& R_{\infty}^{-1}=H_{\infty}=\sqrt{\Omega_{\Lambda}} H_{0}
\end{aligned}
$$

which in turn is determined by gravitational dynamics of the vacuum energy characterized by $\mu$.

$$
\frac{1}{R_{\infty}^{2}}=H_{\infty}^{2}=\frac{8 \pi G}{3} \mu^{4}=\frac{\Lambda}{3} .
$$

We see from the above equations that, despite the formally infinite extent of the spatially flat Friedmann-Robertson-Walker expansion universe, Eq. (2), the presence of a nonvanishing cosmological constant provides a way of ascribing an intrinsic, observer-independent, size parameter to our universe.

In this paper we shall be considering an ensemble of universes similar to our own but with different intrinsic sizes $R_{\infty}$. T The ambivalence between the elementary particle view of the cosmological constant as vacuum energy/pressure and the cosmological view of it as a size parameter for the universe is sharpened by looking at it from this viewpoint. In particular, conventional wisdom would say that all of the basic parameters of the standard model, such as $\Lambda_{Q C D}$ or the electroweak vacuum condensate $v$, are to the best of our knowledge independent of each other. This means that they are also independent of the vacuum energy/pressure characterized by the scale $\mu$, since the cosmological term is just another term in the standard-model Lagrangian density. This in turn implies that $\Lambda_{Q C D}$ and $v$ are also independent of the size $R_{\infty}$ of the universe. By definition this is not the case for the cosmological term ( $c f$. Fig. 1). For universes smaller than our own, the vacuum energy density grows. And for universes

\footnotetext{
${ }^{\dagger}$ Given $\Omega=1$, the only other objective choice of size parameter would seem to be to utilize one of the several landmark times characterizing the history of our universe, e.g. the time of electroweak or strong phase transition, of matter-radiation equality, or of decoupling. Our choice is that of matter-dark energy equality, and appears to us to be the most fundamental.

${ }^{\ddagger}$ Note that $R_{\infty}$ is not the scale-size $R(t)$ characterizing the expansion of our own RobertsonWalker universe. We are not assuming that the fundamental constants are time-dependent. Each universe in the ensemble undergoes its own Big Bang, and is characterized by distinct values of standard-model parameters.
} 
smaller than about $10 \mathrm{~km}$, the vacuum energy density exceeds $1 \mathrm{GeV} / \mathrm{fm}^{3}$, the energy scale of the QCD vacuum.

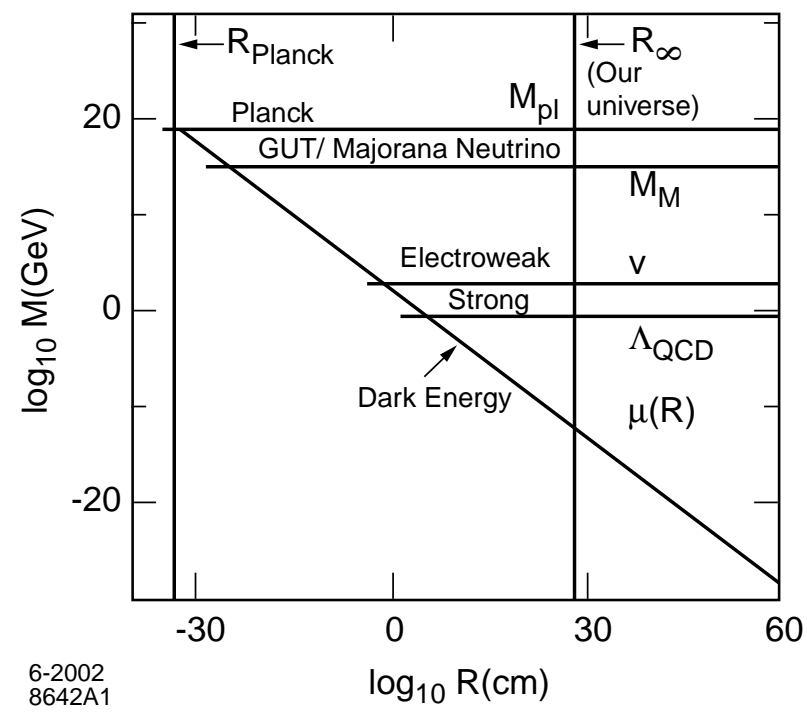

Figure 1: Dependence of fundamental constants on the horizon size $R_{\infty}$ of the universe according to conventional wisdom.

Does this matter at all? It is at least arguable that it does. To really understand the vacuum state is one of the most important goals of fundamental theory, and it is often presumed that one must go to unified theories, such as string theory, to attain true enlightenment. But this would imply that, for the vacuum state, the cosmological degrees of freedom talk to the elementary particle degrees of freedom such as quark/gluon or Higgs in an essential way. This point of view is reinforced by the fact that the dark-energy term in the action is formally renormalized by the quantum corrections contributed by all the other terms in the action.

If there is an interconnection between dark energy and QCD vacuum fluctuations, we might suspect that interesting things occur when the cosmological vacuum energy scale and the QCD vacuum energy scale become comparable. Perhaps there is a discontinuous change, such as occurs for QED at the electroweak scale. Or perhaps the QCD scale does not wait for such a catastrophe to occur, but changes continuously as the size parameter of the universe changes, in a way which is similar to the way 
the cosmological constant itself changes. It is this latter option we entertain in this paper, an option we have in fact already suggested [2]. What we assume is, first, that all dimensionful parameters $X$ of the standard model may vary with $R_{\infty}$, but that to leading approximation they are straight lines in a log-log plot, i.e. they satisfy a simple renormalization-group equation

$$
R_{\infty} \frac{\partial X}{\partial R_{\infty}}=-\frac{1}{2} \mu \frac{\partial X}{\partial \mu}=p_{X} X+\cdots
$$

We shall discuss the unspecified corrections and other details a little more in Section 2.

By itself the above assumption includes the conventional-wisdom option, illustrated in Fig. 田, and contains little news. However we in addition assume that all fundamental dimensional parameters $X$ flow toward a fixed point occurring for universes of approximately Planck/GUT size, and that this is the only such fixed point. Note that only two parameters are needed to describe the gross dependence of $X$ on $R_{\infty}$, and that they are fixed by the value of $X$ at the fixed point and the value of $X$ observed by us. This is illustrated in Fig. 2.

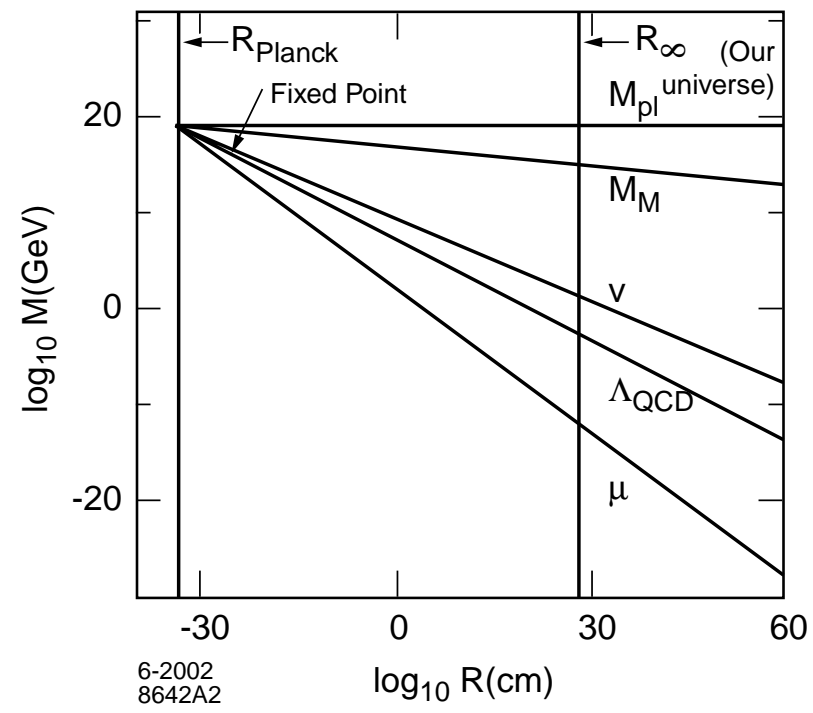

Figure 2: Dependence of the fundamental constants on the horizon size $R_{\infty}$ according to the scaling assumptions. 
The first part of the fixed-point assumption should not be any surprise, since we do expect new physics to occur at the Planck/GUT scale. The second part of the assumption is really what provides the motive power for the remainder of this note, and it is not much more at this stage than an application of Occam's razor. In detail there are probably exceptions to the rule, but perhaps insight can be gained even in the absence of being able to apprehend the exceptions. But leaving aside the possible, even probable complications, what we have at this point is a description of the ensemble of universes we are considering, characterized by the value of $R_{\infty}$, in terms of modified standard-model parameters. And we again emphasize that there are no extra parameters which have been introduced. Therefore we can hope to explore in principle the properties of such universes, using well-defined extrapolations of the laws of chemistry, atomic physics, nuclear physics, etc., with no extra arbitrary assumptions. In particular we can explore the "bandwidth" of features possessed by our own universe. That is, we may try to determine the minimum and maximum sizes $R_{\infty}$ for which nuclear matter exists, or for which hydrogen-burning stars exist, or for which elements as heavy as carbon exist and are produced. These and other examples will be discussed in Section 3, where we shall estimate that if the radius of a universe in our ensemble of universes is within a factor $\sqrt{2}$ of our own, the conditions of life as we know them appear to be satisfied.

Up to this point we may regard the ensemble of universes under consideration as an abstract set, a la Gibbs, and the study of their properties as an abstract intellectual exercise, perhaps of value in the long run in understanding either microphysics beyond the standard model, or the macrophysics of our visible universe. However, there is clear motivation to go further, and to presume that such an ensemble actually exists. In particular we may assume that our universe is one member of a multiverse, with the remaining members causally disconnected from us, as discussed extensively by Rees [3] and others [4].

If such a multiverse ensemble really exists, then a primary quantity of interest is 
the number distribution $n\left(R_{\infty}\right)$ of universes of a given size, defined as

$$
R_{\infty} \frac{d N}{d R_{\infty}} \equiv n\left(R_{\infty}\right)
$$

As mentioned above, we will roughly determine in Section 3 the bandwidth $\Delta R_{\infty} / R_{\infty}$ within which the conditions for life as we understand it exist; it is of order 1 . Then if

$$
\Delta N=n\left(R_{\infty}\right) \frac{\Delta R_{\infty}}{R_{\infty}} \gg 1
$$

we may argue that it is not improbable that life should exist in the multiverse. This is just the condition

$$
n\left(R_{\infty}\right) \gg 1
$$

which appears not to be a heavy constraint. The above line of argument, and concomitant set of problems, parallels the lines of argument used to understand our place in our own universe. Why do we live on Earth rather than Mercury or Pluto? The former is too hot, the latter too cold. Is our existence improbable, in the sense that the parameters characterizing Planet Earth are very finely tuned? The simplest answer is that if life as we know it exists elsewhere in the universe, i.e. there is a sufficiently large population of planets to allow the replication of conditions found on Earth, no fine tuning is required. The jury is still out with respect to what that answer is [5]. But we may argue that the question is, at least in principle, a scientific question. And indeed the hypothesis of a multiverse softens the above constraint to only require that the multiverse contain planets with conditions suitable for supporting life as we know it.

There is an even more specific - and speculative - scenario which can be entertained and which is discussed in Section 5. It is a reductionist version of evolutionary cosmology as envisaged by Smolin [6], utilizing a speculative model of black hole interiors dubbed gravastars by Mazur and Mottola [7, 8]. In this scenario, the interiors of mature black holes are nonsingular and described by the aforementioned static DeSitter metric which characterizes the future of our own universe. This strongly 
suggests a cosmology of nested black holes. The interiors of the black holes in our universe comprise daughter universes, within which there are granddaughter blackhole universes, etc. Going in the opposite direction, we may surmise that our universe consists of the interior of a black hole existing in a mother universe, which in turn is embedded within a grandmother universe, etc. An important issue in this picture of cosmology is the determination of the various species of daughter universes (supermassive galactic-center black holes, stellar-collapse black holes, ...) and their size distribution relative to the size of the parent. Other important parameters are the fertilities of mothers, i.e. the number of daughter universes created per mother, as a function of $R_{\infty}$ and species. We will try to estimate these parameters from data and astrophysical theory, and then try to estimate, for example, the number of sister universes there are in the multiverse, and thereby to re-examine questions posed above, such as estimating the number of planets, galaxies, and/or universes within the multiverse which might support life as we know it.

By now we have clearly entered a highly speculative level. Indeed we have organized this note such that at the beginning of each new section readers making it to that point can become dismissive and bail out. But in the hope that there is at least one person left reading this paragraph, we continue on.

Why all this speculation? From the point of view of this writer, it is motivated by the gravastar scenario, and the related ideas of emergent gravity and emergent standard model, as advocated by Volovik [9] and others [10]. The vacuum is visualized as similar to a quantum liquid such as helium at low temperatures. In the "gravastar" scenario, the blackhole universes are droplets of the quantum liquid, with order parameters which depend on the size of the droplet. The cosmological constant is small because in the ground state of the liquid droplet the pressure (which is measured by the cosmological constant!) vanishes, up to surface corrections. In the picture advocated in this note it is not only the cosmological-constant term in the standard-model Lagrangian density which is a size-dependent order parameter, but all the others as 
well. Indeed for an infinite universe, characterized by an infinite value of the DeSitter horizon radius $R_{\infty}$, the entire standard-model Lagrangian trivializes to a free field theory [2]. All standard-model interactions are therefore viewed as dependent upon the existence of a boundary to our universe. Evidently in the opposite Planck/GUT limit everything becomes strongly coupled.

The crux of this set of ideas lies in the development of a microscopic theory along these lines. And the construction of such a theory may be aided by having a rough picture of the most likely cosmological context for these ideas. It is this which is our primary motivation. But there are a host of obstacles. Some of these are taken up in Section 6, which is devoted to lessons learned and to conclusions, such as they are.

\section{Standard Model Parameters}

The fundamental premise of this paper was already stated above Eq. (17), and we expect this hypothesis to be most accurate for the dimensionful parameters most closely associated with vacuum energy. These are the cosmological-constant scale $\mu$, $\Lambda_{Q C D}$, the electroweak condensate value $v$, and probably a large mass scale associated with neutrino mass, in particular the masses $M$ of the heavy gauge-singlet Majorana particles associated with the see-saw mechanism of neutrino mass generation. These masses appear to be in the range $10^{13}-10^{15} \mathrm{GeV}$, near the GUT scale. For all these quantities, we assume that Eq. (7) holds to good accuracy.

The fact that $\Lambda_{Q C D}$ varies with $R=R_{\infty}$ (hereafter we drop the subscript) leads to an important consequence, namely that the strong coupling constant $\alpha_{s}\left(q^{2}\right)$ must also vary with $R$. Since

$$
\frac{1}{\alpha_{s}\left(q^{2}\right)} \cong b_{s} \ln \frac{q^{2}}{\Lambda_{Q C D}^{2}} \quad b_{s}=\frac{33-2 n_{f}}{12 \pi}
$$

and

$$
\frac{M_{p l}^{2}}{\Lambda_{Q C D}^{2}} \cong\left(M_{p l}^{2} R^{2}\right)^{p_{s}} \quad p_{s} \approx \frac{2}{3}
$$


it follows that

$$
\frac{1}{\alpha_{s}\left(q^{2}, R^{2}\right)}=b_{s} p_{s} \ln M_{p l}^{2} R^{2}-b_{s} \ln \frac{M_{p l}^{2}}{q^{2}} .
$$

What we have is a new renormalization-group equation for $\alpha_{s}$

$$
R^{2} \frac{\partial}{\partial R^{2}}\left(\frac{1}{\alpha_{s}}\right)=b_{s} p_{s}+O\left(\alpha_{s}\right)
$$

which we may compare with the usual expression for the running of $\alpha_{s}$ with $q^{2}$,

$$
q^{2} \frac{\partial}{\partial q^{2}}\left(\frac{1}{\alpha_{s}}\right)=b_{s}+O\left(\alpha_{s}\right)
$$

It is important that this behavior holds for $\alpha_{s}$ evaluated at the GUT scale $M$. As long as coupling constant unification makes any sense at all, we may infer that the electroweak and electromagnetic couplings must also possess the same behavior. Since

$$
\alpha_{s}^{-1}\left(M^{2}, R^{2}\right)=b_{s} \ln \frac{M^{2}}{\Lambda_{Q C D}^{2}} \approx \alpha_{1}^{-1}\left(M^{2}, R^{2}\right) \simeq \alpha_{2}^{-1}\left(M^{2}, R^{2}\right)
$$

it follows that the electroweak couplings at the weak scale are

$$
\alpha_{i}^{-1}\left(v^{2}, R^{2}\right)=\alpha_{i}^{-1}\left(M^{2}, R^{2}\right)+b_{i} \ln \frac{M^{2}}{v^{2}}=b_{s} \ln \frac{M^{2}}{\Lambda_{Q C D}^{2}}+b_{i} \ln \frac{M^{2}}{v^{2}} .
$$

But because $M / v$ and $M / \Lambda_{Q C D}$ scale as powers of $M_{p l} R$, it follows that

$$
\alpha_{i}^{-1}\left(v^{2}, R^{2}\right)=(\text { const }) \ln M_{p l} R \quad i=1,2
$$

and

$$
\frac{\alpha_{1}\left(v^{2}, R^{2}\right)}{\alpha_{2}\left(v^{2}, R^{2}\right)} \equiv \tan ^{2} \theta_{W}=\text { const } .
$$

Therefore the weak mixing angle $\theta_{W}$ is to good approximation independent of $R$, as is the ratio $\alpha / \alpha_{W} \cong \alpha\left(R^{2}\right) / \alpha_{2}\left(v^{2}, R^{2}\right)$. The usual diagram of gauge coupling running and unification is shifted in scale as $R$ is varied. Because the weak mixing angle does not depend upon $R$, the entire figure becomes self-similar (Fig. 3). To leading order, $1 / \alpha$ and $1 / \alpha_{\text {weak }}$ depend linearly on $\ell n R$ and vanish at the Planck/GUT radius (Fig. 本). 

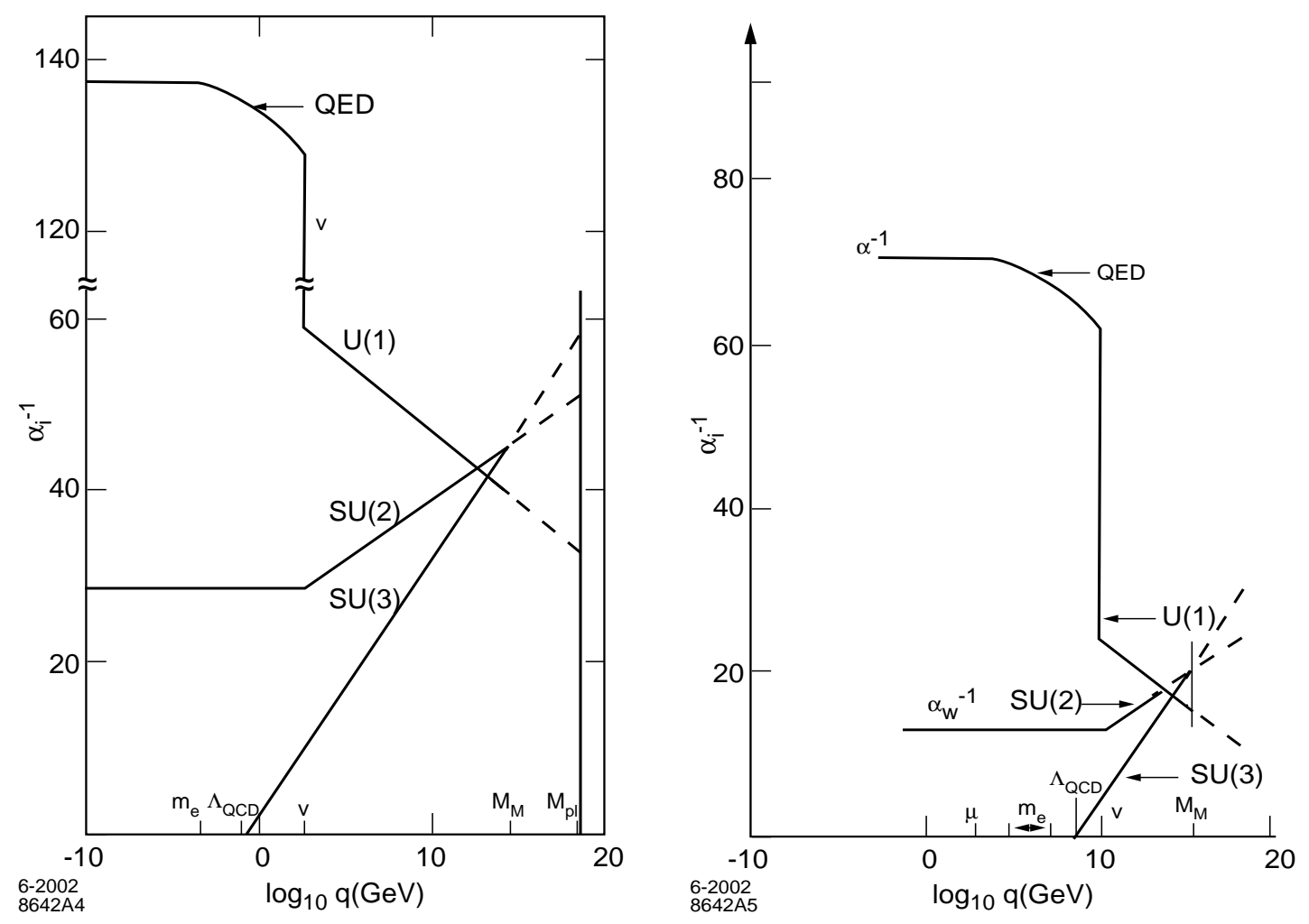

Figure 3: Running of the coupling constants for (a) our universe, and for (b) a universe with horizon size of $10^{-2} \mathrm{~cm}$.

Finally, we shall again invoke Occam and assume that the large Higgs Yukawa couplings $\lambda$ and $h_{\text {top }}^{2}$ are no exception to the rule, and that they also obey the same rule, namely that the inverse couplings vary linearly with $\ell n R$ and vanish for $R$ at the Planck/GUT scale. The usual renormalization group equations connecting the Higgs self-coupling $\lambda$ to the top-quark Higgs coupling $h_{\text {top }}$ (with important QCD corrections) remain unchanged. The new equations are again

$$
R^{2} \frac{\partial}{\partial R^{2}}\left(\frac{1}{h_{t}^{2}}\right)=\text { constant } \quad R^{2} \frac{\partial}{\partial R^{2}}\left(\frac{1}{\lambda}\right)=\text { constant }
$$

and may be used to determine how $\lambda$ and $h_{\text {top }}$, evaluated at either the GUT scale or the infrared scale, vary as $R$ is varied.

We now return to consideration of other standard-model parameters with dimension of mass, starting with the masses of top quark, Higgs bosons, and electroweak 


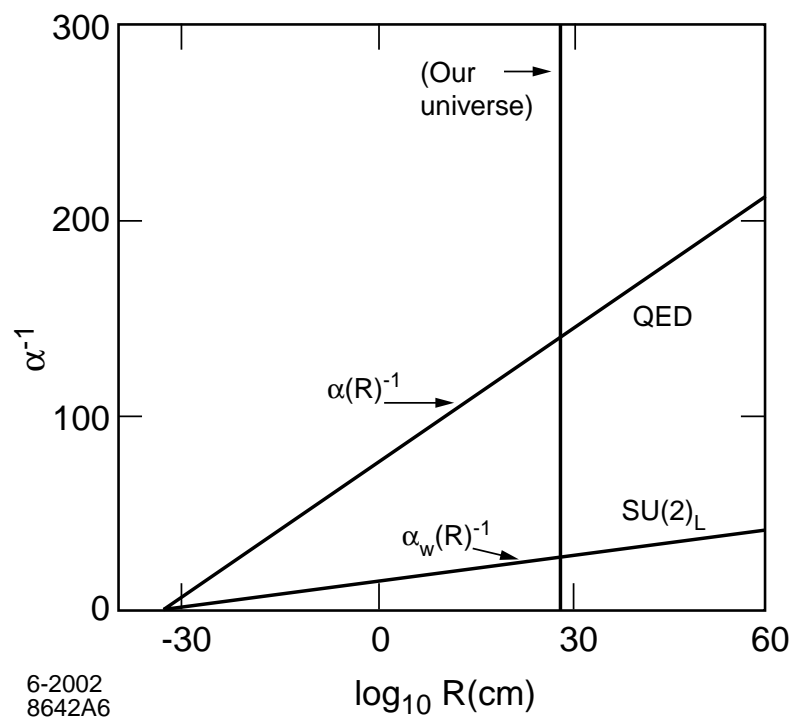

Figure 4: Dependence of $\alpha$ and $\alpha_{\text {weak }}$ (evaluated in the infrared) versus $\log _{10} R$.

gauge bosons, all of which have a mass formula of the form

$$
m=g v
$$

where $g$ stands for a generic dimensionless coupling constant. This implies a renormalization-group equation of the form

$$
\frac{R}{m} \frac{\partial m}{\partial R}=\frac{R}{v} \frac{\partial v}{\partial R}+\frac{R}{g} \frac{\partial g}{\partial R}=p_{v}+(\text { constant }) \cdot g^{2}
$$

which clearly possesses an order $g^{2}$ "radiative correction" to the leading behavior. What is clearly happening is that the ratio $m / v$ is stable, and does not run as a power of $R$ but only as a power of $g$, i.e of $\ell n R$.

In the case of these particles the corrections are not very important, because their masses are so close to the value of the electroweak vev $v$. A more dramatic example is given by the electron mass, which in a sense lies at the opposite extreme. We do not know whether to regard the ratio $m_{e} / v$ as a function of dimensionless coupling constants, i.e. dependent only on $\ell n R$, or as a ratio of fundamental scales, i.e. dependent on a power of $R$. In the former case we have

$$
\frac{v}{m_{e}} \sim(\ln M R)^{n}
$$


where $M$ is the GUT/Planck mass scale. In the latter case the flow is

$$
\frac{v}{m_{e}} \sim(M R)^{p_{e}} \quad p_{e} \approx 0.1
$$

For numerical estimation of the former case we shall choose $n=4$ for the electron. Our motivation is simply to assign one power of some $g^{2}$, of typical order of magnitude, i.e. $(\ell n R)^{-1}$, per mass hierarchy level. Thus for bottom, strange, down, electron, we take $n=1,2,3,4$ respectively.

Returning to the question of $R$-dependence of electron mass, we can evaluate each case. The results are shown in Fig. 5, and we may regard the shaded region as a one-parameter region of uncertainty. We also plot in Fig. 6 the $R$-dependence of the ratio of electron mass to proton mass, which is a crucial parameter for chemistry and condensed matter physics.

The $R$-dependence of other small masses present in the standard model should be similarly regarded, especially the up and down quark masses which drive chiral symmetry breaking of the strong interactions and are responsible for the pion mass. For the strong interactions there is to good approximation only the scale $\Lambda_{Q C D}$, which by itself determines all masses other than that of the pions. In particular the proton mass is proportional to $\Lambda_{Q C D}$, as well as the masses of all mesons and baryons other than the pions (and kaons). But the scale set by the pion mass, whose square varies linearly with the light quark masses and with $\Lambda_{Q C D}$, does matter. It is the $R$ dependence of the ratio of pion to proton mass which will be the crucial parameter for nuclear physics. Its square is plotted in Fig. 8. In Fig. 8, we also plot $\left(m_{K} / m_{\pi}\right)^{2}$, which is proportional to the ratio of strange-quark to down-quark mass.

The remaining parameters of the standard model are $\theta$, the $C P$-violating parameter within QCD, and the CKM mixing parameters, which are closely related to the small quark and lepton masses. In addition there are neutrino masses and mixings. The underlying physics still awaits better understanding, and we have little to add here. These parameters do not appear to be of great importance for what follows in the remainder of this note. 


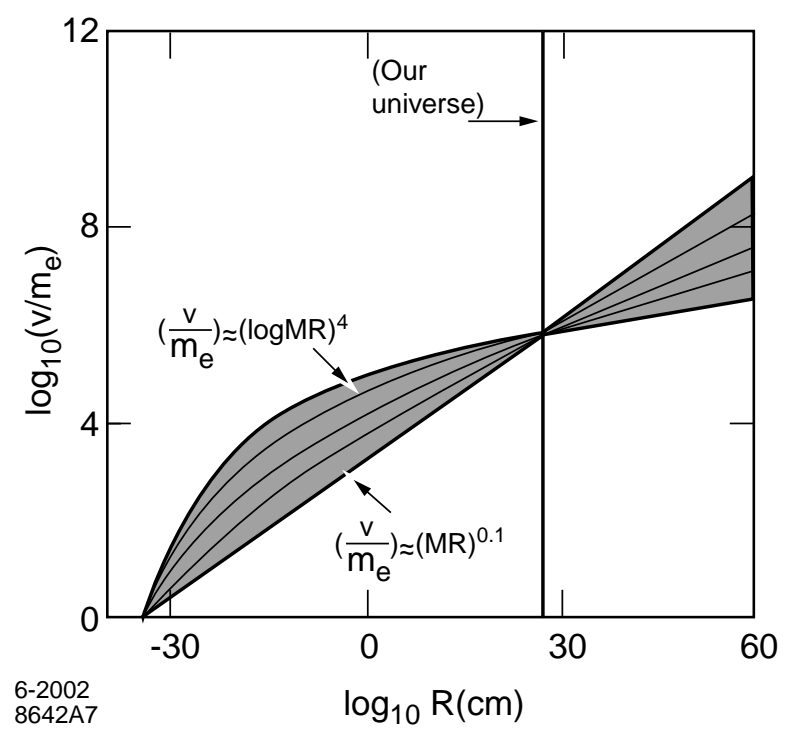

Figure 5: Dependence of the electron mass, scaled to the electroweak vev $v$, versus $\log _{10} R$. The shaded region is a region of uncertainty.

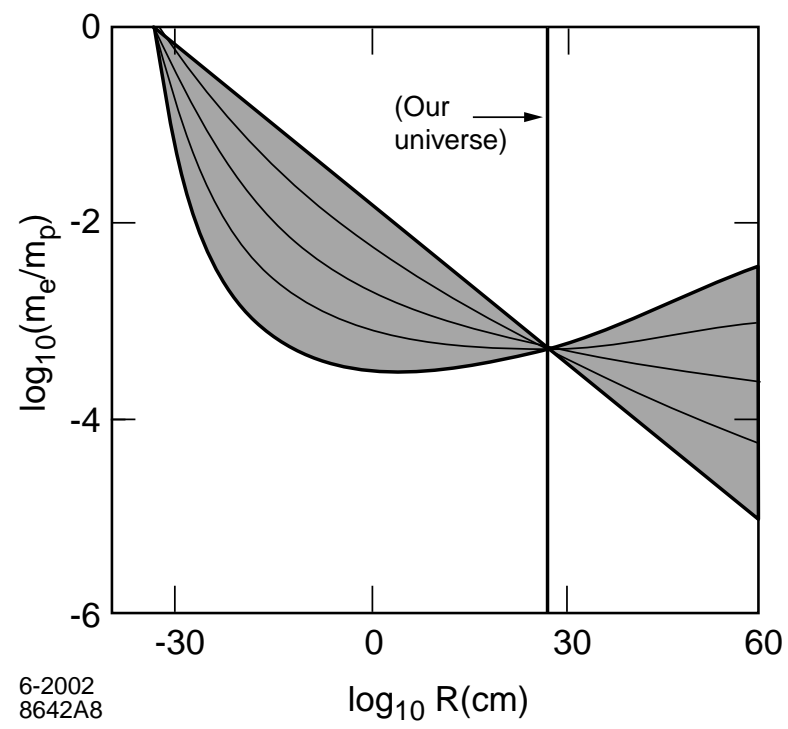

Figure 6: Dependence of $\left(m_{e} / m_{p}\right)$ on $\log _{10} R$. 


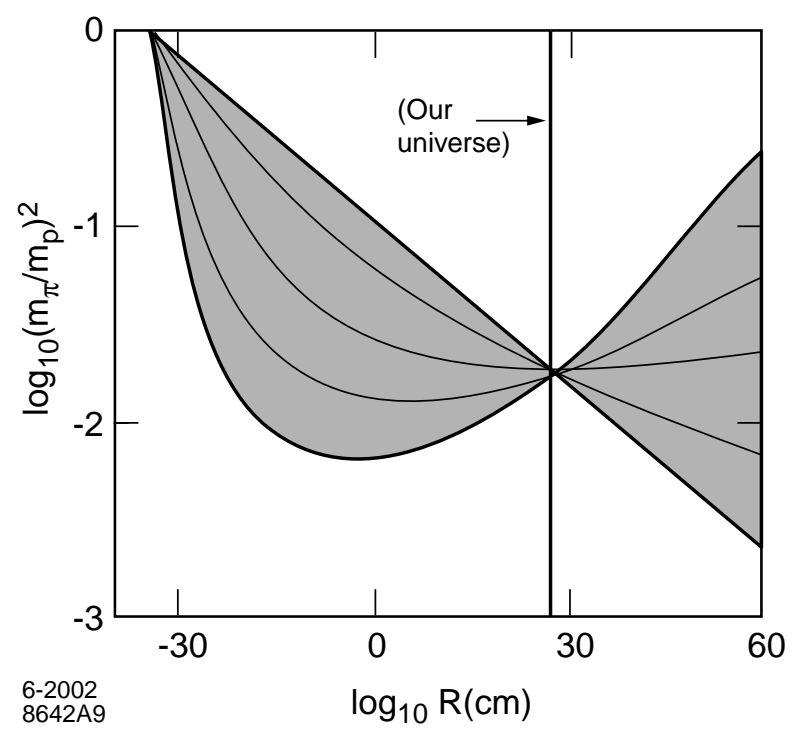

Figure 7: Dependence of $\left(m_{\pi} / m_{p}\right)^{2}$ on $\log _{10} R$.

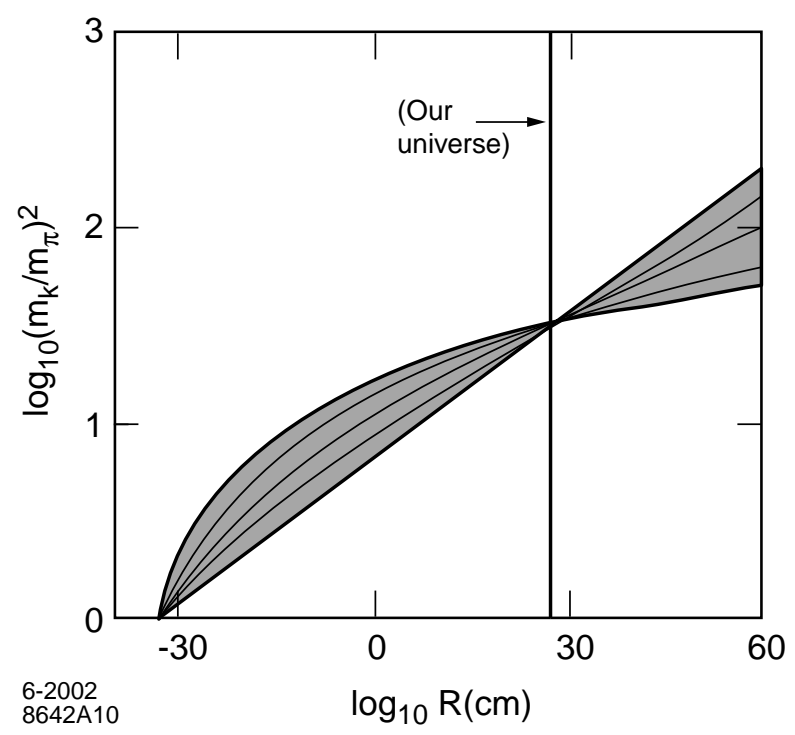

Figure 8: Dependence of $\left(m_{K} / m_{\pi}\right)^{2}$ on $\log _{10} R$ 


\section{Properties of Matter in the Ensemble of Uni- verses}

One of our main goals is to investigate the properties of universes assumed to be almost the same as ours, i.e. with radii $R$ almost the same as ours, and with cosmological initial conditions similar to ours. However we shall also consider more extreme cases, namely universes with radii within 30 orders of magnitude of our own. One reason for considering such large bandwidth is that there will be some properties of these universes which are robust, and do not vary all that much over all those powers of 10. For example the weak and electromagnetic fine-structure constants are in this context robust, having values in this range of $R$ which are within a factor two of what we observe. On the other hand it is well known, especially amongst the "anthropic" community, that other properties of our universe are very finely tuned and will only exist over a quite small bandwidth. We shall pay special attention to such "anthropic" constraints, as discussed for example in the book by Barrow and Tipler [四], and will be interested in the bandwidth in $R$ for which they are satisfied.

We shall begin by considering how the properties of elementary particles, of nuclear matter, and of ordinary matter vary with $R$. We then investigate how the structure of astrophysical objects of interest, such as planets, stars, etc. vary as $R$ is varied.

\subsection{Elementary Particle Properties}

Even for the smallest universe that we shall consider, with radius 100 microns, there is good separation (a factor 10-20) between the electroweak scale and the strong interaction scale. Heavy quarks, electroweak gauge bosons, Higgs particles, etc. are still unstable and will not grossly influence the phenomenology of ordinary matter. A marginal case is that of the strange quark. An estimate of its effect is given by the ratio of kaon to pion mass exhibited in Fig. 8. We see that even for the extreme cases the ratios always stay comfortably above unity, suggesting that we do not err badly 
in neglecting strange-quark contributions to ordinary matter. The strange hadron masses appear to stay high enough to allow semileptonic weak decays at the very least to proceed.

\subsection{Nuclear and Atomic Matter}

Crucial to the properties of nuclear and atomic matter are the values of the finestructure constant (here constrained to a reasonable range of values), the ratio of electron to proton mass, the ratio of pion to proton mass, and the neutron-proton mass difference. As long as the electron-proton mass ratio stays small, atomic physics and chemistry will remain recognizable. We see from Fig. 6 that this is in fact the case. Likewise, in Fig. 7 we see that the pion mass stays well below the proton mass over all the range to be considered.

We conclude that over the 60 orders of magnitude we shall consider, it would appear that chemistry and condensed matter physics will be at least existent and reasonably recognizable. As for nuclear matter, it should exist in recognizable form as the pure chiral limit is approached, because the long range force due to pion exchange is not crucial. It might bind the nucleons a little more (or less), but probably not enough to change the phase structure. However as the pion mass increases, there is more potential for trouble. According to Fig. [7, this appears to occur only in the largest or smallest universes that we shall consider. All this will be discussed in more detail in Section 3.4.

On the other hand, as the pion mass decreases, the neutron-proton mass difference varies in a nontrivial way. It is composed of two pieces. The dominant one is due to the mass difference of the up and down (current-) quarks, and the other is electromagnetic [11]. Schematically we may write

$$
\begin{aligned}
\Delta m=\left(m_{n}-m_{p}\right) & =a_{1}\left(m_{u}-m_{d}\right)+b\left(\alpha m_{p}\right) \\
& =a_{2}\left(\frac{m_{u}-m_{d}}{m_{u}+m_{d}}\right) \frac{m_{\pi}^{2}}{m_{p}}+b\left(\alpha m_{p}\right)
\end{aligned}
$$




$$
\begin{aligned}
& =a_{3} \frac{m_{\pi}^{2}}{m_{p}}+b\left(\alpha m_{p}\right) \\
& \cong 0.1 m_{p}\left[\left(\frac{m_{\pi}}{m_{p}}\right)^{2}-\alpha\right]
\end{aligned}
$$

where we assume that $a_{i}, b$, and the ratio of the difference of up and down quark masses to their sum are to good approximation scale-independent. In the last line we have used the accepted values of the two contributions [11] in approximate form to provide a useful mnemonic. Note that the electromagnetic and quark contributions to $\Delta m$ are of opposite sign. As the chiral limit is approached, the neutron becomes stable and the proton unstable. The latter case is clearly a serious matter for atomic physics and chemistry, which might even cease to exist. However, from Fig. 9 we see that the only cases where this becomes a problem are for universes whose radii are ten to fifteen orders of magnitude larger or smaller than the radius of our universe. The cosmologies for those cases will evidently be nontrivially different from our own, and we will briefly return to this issue later.

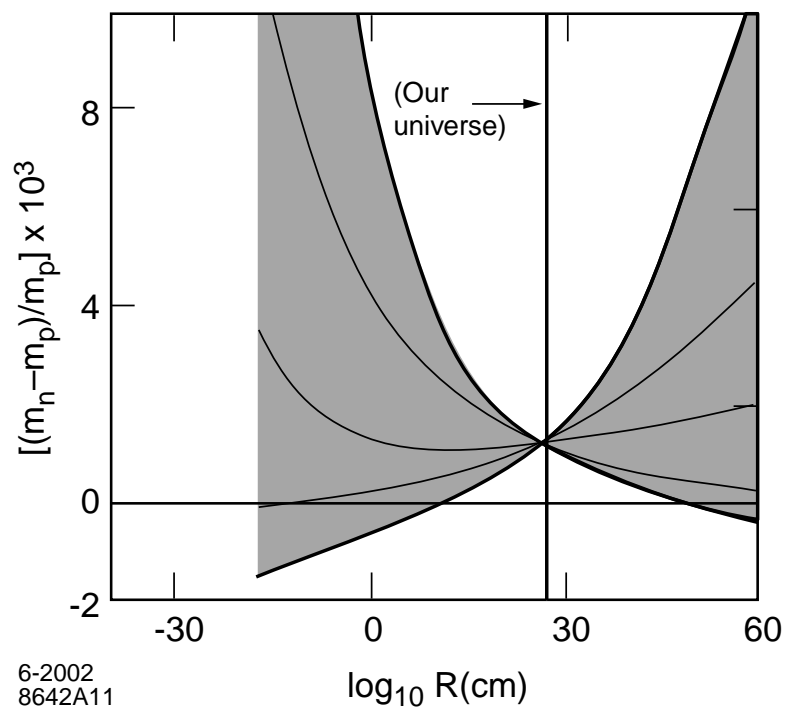

Figure 9: Dependence of the neutron-proton mass difference $\Delta m$, scaled to the proton mass, on $\log _{10} R$. 


\subsection{Stable Cosmological Objects}

Other than black holes, all the large stable cosmological objects exist as a consequence of the Pauli principle. Fermion degeneracy pressure in one form or another provides the repulsion that prevents such objects to gravitationally collapse. This mechanism is so robust that we can expect it to operate over the whole 60 orders of magnitude of radii $R$ which we consider. Three obvious classes to consider are planets, white dwarfs, and neutron stars. In these three cases the degeneracy pressure is provided by nonrelativistic electrons, relativistic electrons, and neutrons respectively. We begin by briefly reviewing these cases.

The density of a planet-like object is fixed by the interatomic force, and the spacing of atomic nuclei is of order $\left(\alpha m_{e}\right)^{-1}$. This gives for the baryon number of a planet of radius $r$ the value

$$
B \cong A\left(\alpha m_{e} r\right)^{3}
$$

where $A$ is the mean atomic number of the nucleus. (If heavy elements are not produced in the universe of interest, then we take $A=1$, and limit our attention to Jupiter-like planets). The chemical binding energy per nucleus is of order the Rydberg, and from this we can determine the total chemical energy and equate it with the gravitational energy in order to determine the characteristic size $r$ of the planet:

$$
U_{\text {chem }} \sim\left(\alpha^{2} m_{e}\right) \frac{B}{A} \sim \frac{B^{2}}{r}\left(\frac{m_{p}}{M_{p l}}\right)^{2} \sim U_{\mathrm{grav}} .
$$

Upon eliminating $r$, this leads to

$$
B \sim \frac{\alpha^{3 / 2}}{A^{2}}\left(\frac{M_{p l}}{m_{p}}\right)^{3}
$$

In a similar way, we may consider white dwarfs, where relativistic electron degeneracy pressure balances the gravitational energy. In that case the baryon number is given by

$$
B \sim r^{3} p_{F}^{3}
$$


where $p_{F}$ is the Fermi momentum of the electron plasma. The energy-balance equation is

$$
U_{\text {degen }} \sim p_{F} B \sim \frac{B^{2}}{r}\left(\frac{m_{p}}{M_{p l}}\right)^{2} \sim U_{\text {grav }}
$$

which simplifies to

$$
B \sim\left(\frac{M_{p l}}{m_{p}}\right)^{3} .
$$

Finally, we may consider the case of the neutron star. It is similar to the white dwarf case. One simply replaces the electron Fermi momentum $p_{F}$ with $\Lambda_{Q C D}$ which characterizes the neutron Fermi momentum, and arrives at the same result.

We see that in all three cases the baryon number, hence the mass, of the object scales as the inverse third power of the proton mass, and therefore scales as the appropriate power of $R$. The result is shown in Fig. 10. We therefore can anticipate the existence and can understand the properties of these massive objects, throughout the 60 orders of magnitude of $R$ we consider. Stars, however, are another matter. The question of whether these large objects ignite and burn, and for how long, depends on details. Before addressing stellar structure we consider some of the finer points having to do with the nuclear force.

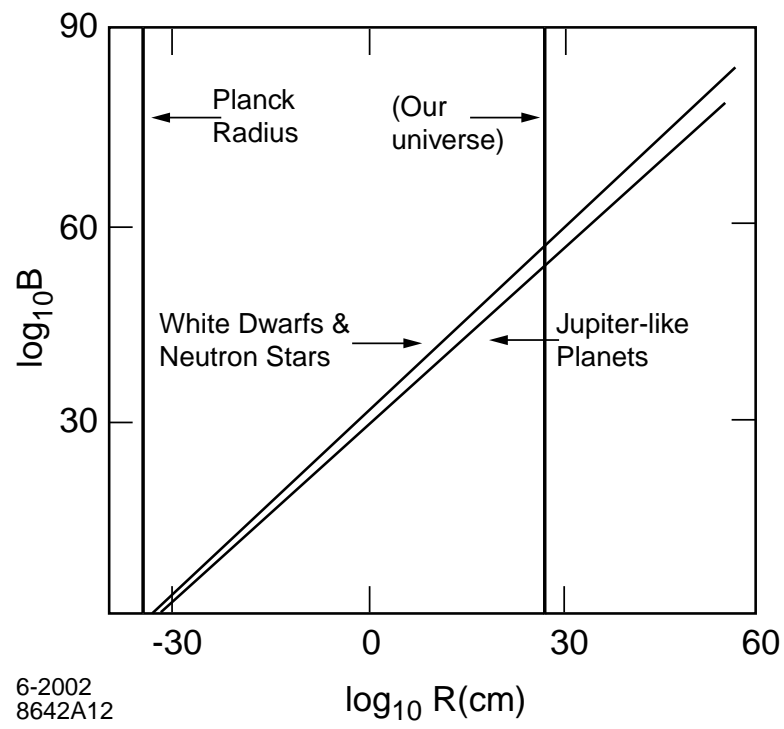

Figure 10: Dependence of baryon number $B$ of astrophysical objects upon $\log _{10} R$. 


\subsection{The Nuclear Force}

The simplest system in nuclear physics is the dinucleon. It is a delicate case, especially in the context of astrophysics, because the nonexistence of bound diprotons and/or dineutrons is needed to keep stars burning [4, [12], and the existence of a deuterium bound state is an essential ingredient for fusion reactions in stars as well as in bigbang nucleosynthesis. What is needed is the dependence of the binding energies upon $\left(m_{\pi} / m_{p}\right)$. There is no consensus on what the answer is. Depending upon the method, different magnitudes and even signs are obtained [13, 14, 15]. What is important in our application is the value of $\left(m_{\pi} / m_{p}\right)$ for which the deuteron becomes unbound, as well as the value of $\left(m_{\pi} / m_{p}\right)$ for which the diproton and/or dineutron might become bound. We choose here an estimate which lies in the midrange of what is generally considered [15, 16], and has the sign dictated by naive intuition; as one approaches the chiral limit, the binding energies increase. Our choices are as follows:

$$
\begin{aligned}
& \text { Deuteron bound if } m_{\pi} / m_{p} \leq 0.16 \\
& \text { Diproton bound if } m_{\pi} / m_{p} \leq 0.08 .
\end{aligned}
$$

We emphasize that these choices are uncertain, but probably by not more than a factor 3. However, it is arguable [15, 16] that the dinucleon remains unbound even in the chiral limit, in contradiction to the choice made above. But, it will turn out that in what follows we will not consider any region of parameter space where, given the parameters we have chosen, the diproton is bound, so that for us the issue is moot.

Finally, we may consider the mechanism for producing carbon in stars. This depends upon the existence of the anthropically famous triple- $\alpha$ reaction [17]

$$
\begin{aligned}
& { }^{4} \mathrm{He}+{ }^{4} \mathrm{He} \rightarrow{ }^{8} \mathrm{Be} \\
& { }^{8} \mathrm{Be}+{ }^{4} \mathrm{He} \rightarrow{ }^{12} \mathrm{C}+2 \gamma
\end{aligned}
$$

with the resonance in ${ }^{12} \mathrm{C}$ predicted by Hoyle [18], together with the absence of a crucial level in ${ }^{16} O$. The parameter sensitivity of this process, which is of order $\delta E \sim$ 
$100 \mathrm{keV}$ in a system with binding energy scales in the $10 \mathrm{MeV}$ range, is discussed by Oberhummer et al. [19], among others [20]. The result is that an 0.3 percent variation in the overall strength of the nuclear force is enough to strongly modify this delicately balanced mechanism. If such a perturbation were applied to the deuteron, it would change its binding energy by about five percent. We conclude that at most the triple- $\alpha$ process represents a parameter sensitivity a factor 20 greater than what one obtains from considering dinucleon binding. However, the actual sensitivity of the triple alpha mechanism may be considerably less, because the dinucleon binding could be more sensitive to the long-range pionic tail of the force than the interactions between compact, closed-shell alpha-particles. It would be helpful to have a good description of the dependence of the intermediate-range, isosinglet, spin-independent attractive force upon pion mass. But at present this seems not to exist.

\section{Cosmology}

In this section we explore how big bang cosmological evolution depends on the ultimate "size" $R$ of the particular universe which is created. There are a variety of epochs in the history of a universe which are especially sensitive to parameter variations. Before going into more details we briefly sketch them here to set the stage:

1. We take as initial condition of the universe its state just after inflationary reheating (the assumption that inflation indeed occurs will not be too important), with the initial temperature taken to be of the Planck/GUT scale. The universe is always taken to be spatially flat. The magnitude of the primordial density fluctuations, which eventually account for the observed fluctuation spectrum in the 3 degree microwave background, is in principle a parameter to be specified. In practice we shall choose it to be equal to what it is in our universe, $\delta_{H}=\delta \rho / \rho \sim 2 \times 10^{-5}$, independent of $R$.

2. The baryon asymmetry of the universe is assumed to be generated in some 
intrinsic way from unknown, extended-standard-model mechanisms at a very high temperature scale. The details of this mechanism are at present very uncertain. Therefore the $R$ dependence of this asymmetry will be treated in a way similar to how the electron mass was treated. We assume that for universes with a size of order the Planck radius, the baryon asymmetry is large, of order unity. We assume that the interpolation from the Planck size to large universes like our own may behave as a power of $R$, or as a power of $\ell n R$, each option taken to be an extreme case. The result is shown in Fig. 11. While the uncertainties become large for universes very different in size from our own, at least the dependence upon $R$ is monotonic.

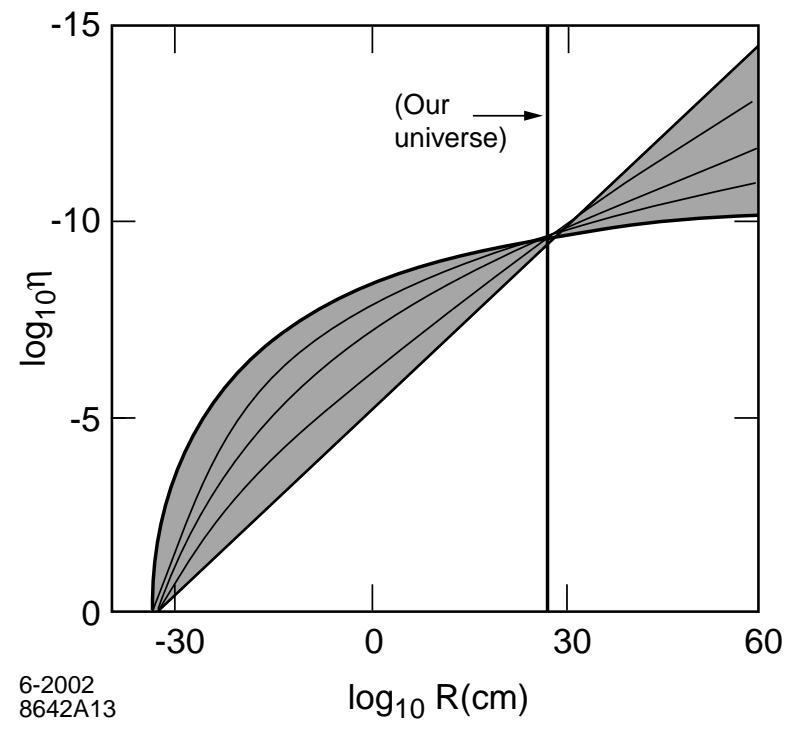

Figure 11: Conjectured dependence of the baryon-to-photon entropy ratio $\eta$ upon $\log _{10} R$.

3. As the universe cools, the phase transitions at electroweak and QCD scales proceed in a way similar to our universe. (The baryon asymmetry may be modified at the electroweak scale via "sphaleron" effects [21], and if so it is the modified asymmetry which is shown in Fig. 11.) Differences appear at the epoch of nucleosynthesis, at a temperature of order $10^{-3} m_{p}$. The mechanisms are sensitive to the time at which neutrinos decouple from the plasma and baryonic chemical equilibrium is lost. The abundances of ${ }^{4} \mathrm{He}$, deuterium, and hydrogen (and even ${ }^{2} \mathrm{He}$ ) become sensitive to the 
parameters and require a detailed discussion.

4. At some very uncertain temperature scale, (cold) dark matter decouples from the plasma and evolves, eventually becoming a major component of the matter density. The physics of this is obscure. We shall assume that the cold dark matter is composed of WIMPs, by which we mean that their interactions with each other and with ordinary matter are characterized by a scale somewhere around the electroweak scale.

5. The epochs of matter-radiation equality and of decoupling of radiation from matter are also parameter sensitive. What happens during these periods provide initial conditions for the subsequent evolution of large-scale structure formation. All of this will require a detailed discussion.

\subsection{Nucleosynthesis}

As the universe cools below the QCD phase transition, quarks and antiquarks bind into mesons and baryons, and the mesons soon disappear. Neutrons and protons are kept in chemical equilibrium by electroweak scattering processes induced by neutrinos. Eventually the neutrinos decouple, the criterion for decoupling being that the expansion rate of the universe exceed the collision rate. The expansion rate for a radiation dominated universe is

$$
H^{2} \sim \frac{T^{4}}{M_{p l}^{2}} .
$$

Equating $H$ to the collision rate gives

$$
H \sim \frac{T^{5}}{v^{4}} .
$$

This leads to the criterion

$$
\left(\frac{T}{m_{p}}\right) \sim\left(\frac{v}{m_{p}}\right)\left(\frac{v}{M_{p l}}\right)^{1 / 3} .
$$

This result is plotted in Fig. 12 . 


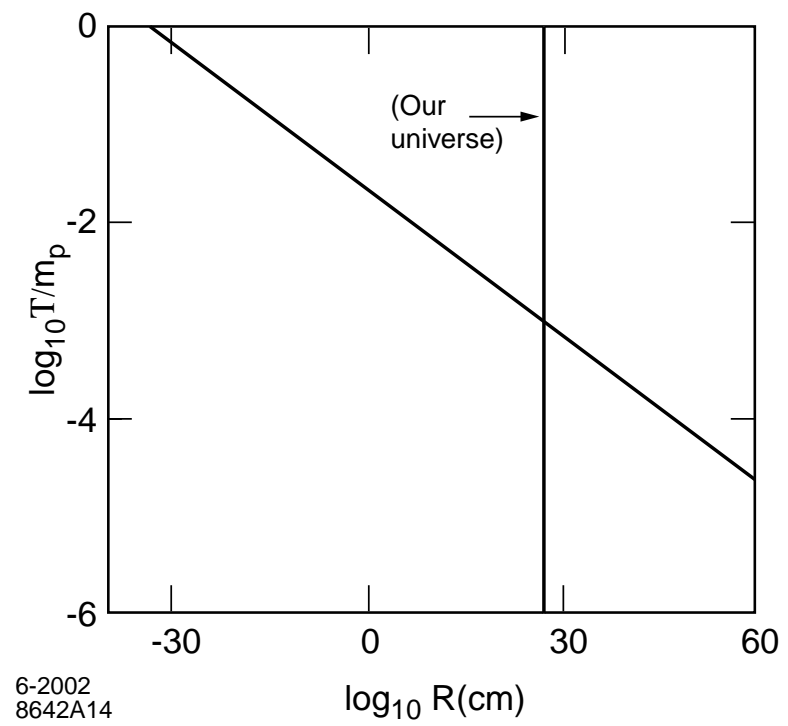

Figure 12: Dependence of the temperature at which neutrinos decouple from matter, scaled to the proton mass, upon $\log _{10} R$.

In our universe, neutrino decoupling occurs at a temperature of about $0.7 \mathrm{MeV}$. At that temperature the ratio of neutrons to protons has been depleted by about a factor seven, due to the Boltzmann factor containing the neutron-proton mass difference. The remaining neutrons capture into deuterium, which is then converted quickly to ${ }^{4} \mathrm{He}$ by fusion reactions. The net result is a primordial helium abundance of 22 percent or so.

Had neutrino decoupling occurred much earlier, the neutron-proton ratio would have been unity. All the baryons would end up as deuterium, which would then convert via fusion to helium. Conversely, if decoupling were to occur much later, then the neutrons would be removed by the neutrino reactions, and there would be nothing left at low temperatures but hydrogen. We therefore expect the dependence of the abundance of primordial helium to change from very high for $R$ smaller than the radius of our universe to very low for $R$ greater.

However, for very large or small $R$ the situation is more complicated and in fact uncertain. As $R$ varies the ratio of pion to proton mass varies, and with it the binding energy of the dibaryons. For large values of $\left(m_{\pi} / m_{p}\right)$, the deuteron probably does not 
exist, and the fusion reactions are blocked. If the pion mass is very small, then the diproton may be bound, and instead of hydrogen in the final state of proton universes, there would initially be ${ }^{2} \mathrm{He}$. Further complicating the situation is the $R$ dependence of the ratio of neutron-proton mass difference to electron mass, which for small $R$ can fall below unity, leading to a stable neutron. In addition, if $\left(m_{\pi} / m_{p}\right)$ is sufficiently small, the neutron-proton mass difference changes sign. The situation is sketched out in Fig. 13, where we identify various regions in the two-dimensional parameter space of $R$ and $\left(m_{\pi} / m_{p}\right)$ for which the baryogenesis scenarios qualitatively change. There are seven distinct regions of the parameter space we consider. Regions I, IV, and V are characterized by an unstable deuteron. Regions V, VI, and VII are characterized by a stable neutron. In most of region VII the proton is unstable. In regions I and II neutrinos decouple at such a low temperature that the fraction of neutrons in the mix is less than 5 percent. Consequently those universes evolves into predominantly hydrogen. In region VII the opposite occurs, and the ${ }^{4} \mathrm{He}$ fraction exceeds 90 percent. Only in region III is the situation qualitatively the same as for our universe. If $R$ lies between $10^{24} \mathrm{~cm}$ and $10^{33} \mathrm{~cm}$, this is assured to be the case, although this conclusion rests heavily upon the assumption made in Eq. (32).

We now briefly describe the individual baryogenesis histories for the seven regions we have identified:

Region I: In this region the deuteron is unbound, and the decoupling of neutrinos occurs so late that the $n / p$ ratio is less than 5 percent. The result is a nearly pure hydrogen universe. However fusion reactions within stars will not proceed because of the absence of deuterium.

Region II: Again the $n / p$ ratio is less than 5 percent, and a predominantly hydrogen universe is formed. But now the deuteron exists, so that in principle stars can burn hydrogen into helium.

Region III: As noted above, this region resembles - and includes-our own universe. 


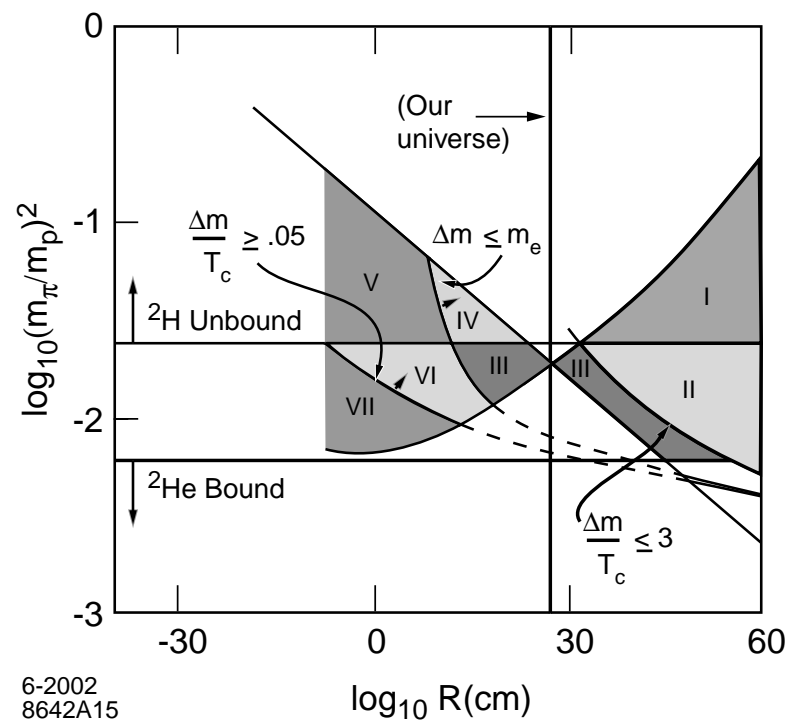

Figure 13: Regions of parameter space for which cosmological evolution is qualitatively different. See the text for the details.

Region IV: In this region the deuteron is unbound. Although neutrinos decouple relatively early, when the $n / p$ ratio is not too small, the fusion reactions are blocked. The extra neutrons decay, and we are again left with a hydrogen-dominated universe. But as in Region I, fusion reactions within stars are blocked.

Region V: This region differs from Region IV, because here the neutron is stable. The universe will be mixed hydrogenic and neutron, with fusion reactions again blocked because of the absence of deuterium.

Region VI: In this region, deuterium exists. The $n / p$ ratio at neutrino decoupling is not small, so that nucleosynthesis of ${ }^{4} \mathrm{He}$ should proceed. The neutron is stable, but primordial neutrons are presumably found in the helium. Fusion reactions in stars should be able to proceed.

Region VII: In most of this region the proton is unstable and decays to the neutron with positron emission. The $p / n$ ratio is large enough (but less than unity!) so that primordial ${ }^{4} \mathrm{He}$ will be produced. Because the deuteron is stable, fusion reactions in stars may proceed. Hydrogen-based chemistry will not exist, although perhaps some deuterium-based chemistry might survive. 
It is noteworthy that in all seven regions the electron chemical potential does not vanish. An electron plasma will persist until decoupling occurs at a much lower temperature scale.

\subsection{Stars}

The properties of these regions are perhaps well enough defined that one could go further and map out the subsequent cosmological history in a little more detail. We shall not try to do so here. But before going on to more general cosmological questions, we will consider (within our Region III) the additional oft-cited constraints on the existence of long-lived stars and of the conditions appropriate to the production of carbon and other heavier elements [12, [4, 6]. An immediate reason for doing so is anthropic; we would like to know the bandwidth in $R$ within which the changes in standard model parameters are small enough to preserve the conditions in our universe which are conducive to life as we know it.

Quite a long list of "anthropic" constraints exist. Upon examination of the items on that list, it should come as no surprise that the most restrictive by far is the existence of the triple- $\alpha$ fusion-reaction chain which allows the production of carbon and thereby the existence of heavier elements. We already mentioned in Section 3 that this constraint could be 20 times more sensitive than the constraints used above regarding the existence of bound deuterium. Examination of Fig. 13 shows that this enhanced sensitivity roughly translates into

$$
\left|\log _{10} \frac{R}{R_{0}}\right|<0.2
$$

or that

$$
0.7<\frac{R}{R_{0}}<1.5
$$

where $R_{0}$ is the radius of our universe. In other words, if the radius of a universe in our ensemble of universes is within roughly a factor $\sqrt{2}$ of ours, the standard model 
parameters are close enough to our own not to upset the conditions necessary for existence of life in that universe.

\subsection{Large Scale Structure}

As the universe continues to cool and expand, the era of matter dominance emerges. In the scenario we consider $(\Lambda C D M)$, it is the cold dark matter that is essential in initiating the growth of density fluctuations. The baryons carry less of the energy density, and they stay coupled to the photons for much longer, thereby being unable to fully participate in the growth of inhomogeneities until decoupling is reached.

We review briefly the standard calculations in order to see the parameter dependencies [21]. The abundance of cold dark matter WIMP particles $X$ is estimated by equating their rate of production and/or annihilation to the Hubble expansion rate at the time/temperature of WIMP decoupling.

$$
n_{X}\langle\sigma v\rangle \sim H \sim \frac{T^{2}}{M_{p l}} .
$$

Here $n_{X}$ is the number density and $T$ the temperature at decoupling. Normalizing the abundance to the abundance of photons, proportional to the cube of the temperature, gives

$$
\left(\frac{n_{X}}{n_{\gamma}}\right) \sim \frac{1}{M_{p l} T\langle\sigma v\rangle} \sim \frac{20}{m_{X} M_{p l}\langle\sigma v\rangle}
$$

where we use the fact that within a factor two the WIMP decoupling temperature is

twenty times lower than the rest mass of the $X$ particles over a very wide range of parameters [21].

We may further relate this to the abundance of baryons, by introducing the baryon-to-photon entropy ratio $\eta$, already discussed above and depicted in Fig. 11:

$$
\frac{\Omega_{X}}{\Omega_{B}} \sim\left(\frac{m_{X}}{m_{p}}\right)\left(\frac{n_{X}}{n_{\gamma}}\right)\left(\frac{n_{\gamma}}{n_{p}}\right) \sim \frac{20}{m_{p} M_{p l}\langle\sigma v\rangle \eta} .
$$

When this is evaluated for our universe, the cross section estimate is

$$
\langle\sigma v\rangle \sim\langle\sigma\rangle \sim 10^{-37} \mathrm{~cm}^{2}
$$


This cross section is close to the electroweak scale. Therefore we assume, as do many others [22], that it scales with the inverse square of the electroweak vev $v$ and perhaps some power of a coupling constant,

$$
\langle\sigma v\rangle \sim \frac{\alpha^{n}}{v^{2}}
$$

with $n$ taken to be two or three.

We may now look at the $R$-dependence of the ratio of dark matter to baryonic matter. It is plotted in Fig. 14. We see that dark matter will dominate over baryonic matter provided the radius of the universe is greater than $10^{-4}$ of our own.

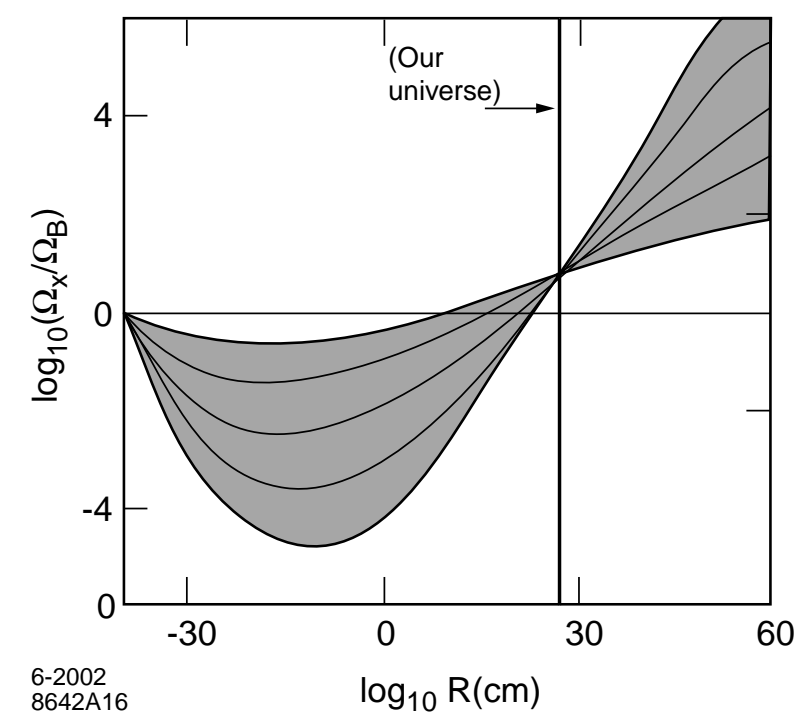

Figure 14: Estimated ratio of dark matter to baryonic matter $\Omega_{X} / \Omega_{B}$ as a function of $\log _{10} R$.

We may also determine the temperature $T_{E Q}$ when the contributions of matter and radiation are equal, and which signals the onset of matter-dominated expansion of the universe. From Eq. (40) we find

$$
\frac{\rho_{X}}{\rho_{\gamma}} \sim \frac{m_{X}}{T}\left(\frac{n_{X}}{n_{\gamma}}\right) \sim \frac{20}{T M_{p l}\langle\sigma v\rangle} \sim \frac{20 v^{2}}{T M_{p l} \alpha^{n}}
$$

from which it follows

$$
T_{E Q} \sim 20 \frac{v^{2}}{M_{p l} \alpha^{n}} .
$$




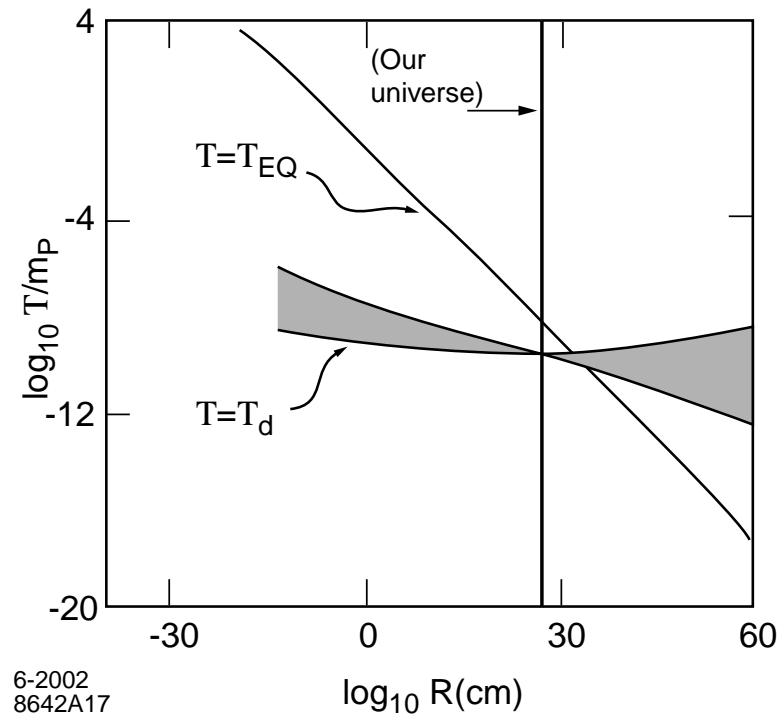

Figure 15: Temperature $T_{E Q}$ for which nonrelativistic matter and radiation are equal as a function of $\log _{10} R$. Also shown is the temperature $T_{d}$ at which matter and radiation decouple, versus $\log _{10} R$.

This is plotted in Fig. 15. Also shown there is the temperature at which radiation decouples from ordinary matter and the universe becomes transparent. The formula which controls this is [21]

$$
T_{d} \sim \frac{\alpha^{2} m_{e}}{40}
$$

We see that if $R$ is less than $10^{5}$ the size of our universe

$$
T_{d}<T_{E Q}
$$

while the opposite is true for larger universes. Therefore for small universes the WIMP degrees of freedom will first feel the Jeans instability, with the growth of fluctuations in baryonic matter occurring later. For the large universes the baryonic fluctuations grow together with the WIMP fluctuations. It is not immediately clear how much of a difference this might make in the creation of large-scale structure.

The evolution of the large scale structure is in general a complex topic. The most straightforward part of the subject consists in the growth of small density perturbations in the linear regime. As mentioned above, we assume that the typical scale of 
primordial perturbations, present at the earliest epoch we consider when temperatures were at the Planck/GUT scale, are of order

$$
\left(\frac{\delta \rho}{\rho}\right)_{0} \sim 2 \times 10^{-5}
$$

as measured by the cosmic microwave background temperature fluctuations. These density perturbations remain frozen at more or less this value during the radiationdominated epoch, but grow rapidly once the matter-dominated epoch begins, provided their wavelength is less than the horizon scale at the time $t_{E Q}$ of matterradiation equality. The growth of the amplitude scales with the scale factor of the universe

$$
\left(\frac{\delta \rho}{\rho}\right)_{R} \sim\left(\frac{R}{R_{E Q}}\right)\left(\frac{\delta \rho}{\rho}\right)_{0}
$$

provided the perturbation is small and one remains in the linear regime. After matter dominates radiation, the Robertson-Walker scale factor of the universe is given by

$$
R(t)=R_{0}\left(\sinh \frac{3}{2} H_{\infty} t\right)^{2 / 3}
$$

When the sinh factor equals unity, one has equal amounts of ordinary (dark plus baryonic) matter and dark energy. We denote this point in time with a subscript $\Lambda$

$$
\sinh \frac{3}{2} H_{\infty} t_{\Lambda}=1
$$

and define this as "cosmological freezeout." For later times, when dark energy is dominant, the growth of fluctuations will cease, and again be frozen in place. Our own universe is in this state of transition, with the present time $t_{0}$ given by

$$
\tanh \frac{3}{2} H_{\infty} t_{0}=\sqrt{\Omega_{\Lambda}} \approx 0.84
$$

It will in general suffice to equate the present time $t_{0}$ with $t_{\Lambda}$.

The total amount of growth of initial perturbations, from matter-radiation equality to late times, is therefore simply given (assuming linearity) by the redshift factor 
between $t_{E Q}$ and $t_{\Lambda}$. Putting in the numbers for our universe, for short-wavelength modes, one finds

$$
\left(\frac{\delta \rho}{\rho}\right)_{\Lambda} \sim\left(\frac{R_{\Lambda}}{R_{E Q}}\right)\left(\frac{\delta \rho}{\rho}\right)_{0}=(1+z)_{E Q}\left(\frac{\delta \rho}{\rho}\right)_{0} \sim\left(\frac{T_{E Q}}{T_{\Lambda}}\right)\left(\frac{\delta \rho}{\rho}\right)_{0} \sim\left(3 \times 10^{4}\right)\left(\frac{\delta \rho}{\rho}\right)_{0} .
$$

This marginally contradicts the linearity assumption. Therefore for fluctuations of wavelength large compared to the critical wavelength $\lambda_{E Q}$, the total amount of growth will remain in the linear regime. Consequently we expect that the largest structures exhibiting very high density contrast will be limited in size to roughly $\lambda_{E Q}$, defined as the wavelength or frequency (in comoving conformally flat coordinates) which is comparable to the horizon scale at $t_{E Q}$. Since

$$
\lambda \sim \int_{0}^{t} \frac{d t^{\prime}}{R\left(t^{\prime}\right)}=\frac{3 t}{R(t)} \sim t^{1 / 3} \sim R(t)^{1 / 2}
$$

it follows that the physical size of this structure at present is

$$
r_{0}=R_{0} \lambda_{E Q}=\left[\frac{12 t_{E Q}}{H_{\infty}^{2}}\right]^{1 / 3} \cong H_{\infty}^{-1}\left(\frac{8 t_{E Q}}{t_{\Lambda}}\right)^{1 / 3}=\frac{8^{1 / 3} H_{\infty}^{-1}}{(1+z)_{E Q}^{1 / 2}}
$$

which scales as the inverse square root of the redshift factor, as shown. For our universe, this implies that the density contrast should be small on scales larger than about $1 / 400$ of the size of the universe, characterized above by $H_{\infty}^{-1}$, and large on scales smaller than that. This is consistent with what is observed.

As we mentioned above, we expect that these gross features of this structure formation are (for the $\Lambda C D M$ scenario) determined by the cold dark matter which is the dominant component of the matter density. On the other hand, the structure on smaller scales may crucially depend upon the baryonic component of the ordinary matter, because, according to our WIMP hypothesis, the dark matter component acts as a collisionless dilute gas, while the baryonic component is more susceptible to nongravitational dissipative mechanisms.

We may now investigate how much things change as the radius $R$ of the universe is varied. We have assumed (quite arbitrarily) that the primordial fluctuation scale 
is $\sim 2 \times 10^{-5}$ independent of $R$. As described above, this fluctuation in general grows linearly with $R$ from the time $t_{E Q}$ of matter-radiation equality until the time $t_{\Lambda}$ at which dark matter and dark energy (cosmological constant) contribute equally to the Hubble expansion. The former is given in Fig. 15 and Eq. (45), while the latter is given by Eq. (51).

$$
T_{E Q} \sim \frac{20 v^{2}}{M_{p l} \alpha^{n}} \quad t_{\Lambda} \sim H_{\infty}^{-1} \equiv R
$$

We must convert $T_{E Q}$ to $t_{E Q}$ using the fundamental relationship between them, valid in the radiation-dominated epoch:

$$
t_{E Q} \sim \frac{M_{p l}}{T_{E Q}^{2}}
$$

We thereby obtain

$$
\left(\frac{\delta \rho}{\rho}\right)_{\Lambda} \sim\left(\frac{t_{\Lambda}}{t_{E Q}}\right)^{2 / 3}\left(\frac{\delta \rho}{\rho}\right)_{0} \sim\left(\frac{R T_{E Q}^{2}}{M_{p l}}\right)^{2 / 3}\left(\frac{\delta \rho}{\rho}\right)_{0} \equiv(1+z)_{E Q}\left(\frac{\delta \rho}{\rho_{0}}\right)
$$

The size $r_{0}$ of the largest structures, relative to the size $R$ of the universe, follows from Eq. (55) and has a similar form:

$$
r_{0} \sim\left(\frac{t_{E Q}}{t_{\Lambda}}\right)^{1 / 3} R \sim\left(\frac{R T_{E Q}^{2}}{M_{p l}}\right)^{1 / 3} R \equiv(1+z)_{E Q}^{-1 / 2} R
$$

The $R$ dependence of the red-shift factor $(1+z)_{E Q}$ is shown in Fig. 16. We see that for a rather large bandwidth, of order 10 to 15 powers of ten, the amplification of the primordial perturbations is within an order of magnitude of what is present in our own universe. We also recall that for universes larger than $10^{-4}$ of ours, the baryonic fraction $\Omega_{B} / \Omega_{X}$ is small, but not negligibly so. Therefore we may surmise that, whatever the mechanism is that creates black holes in the centers of galaxies, it will probably still be operative in this class of universes as well. However, the basis for this conclusion is very fragile, since it rests upon our assumptions of the properties of dark matter. 


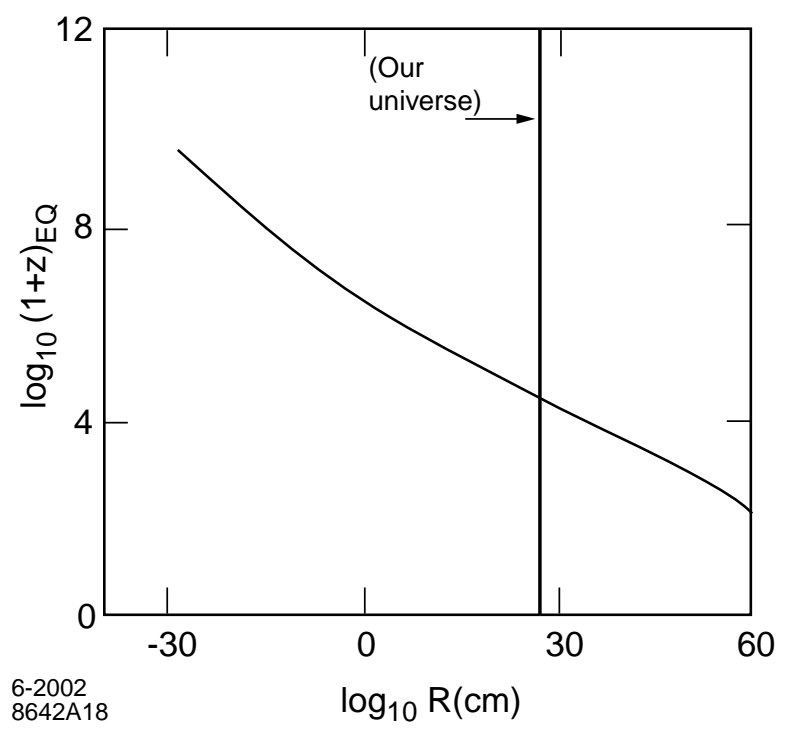

Figure 16: Dependence of the amplification of primordial fluctuations, $R_{\Lambda} / R_{E Q}=$ $(1+z)_{E Q}$ upon $\log _{10} R$.

\section{Emergent Cosmology}

If the ensemble of universes we have been considering actually exists, then there are anthropic consequences, as mentioned in the introduction. From the behavior found in the previous section, we may conclude that if the number of universes per octave (factor two) in radius $R$ is large compared to unity, then it is reasonable that we should be present in the ensemble [23]. There is a caveat; if the mean number of planets per universe (of our size $R$ ) which are appropriate for the support of life as we know it is small compared to unity, then the number of universes per octave needed to make reasonable our existence must be correspondingly increased. The planetary situation is not well understood [5], so this option is not academic. But either way, it would not seem outrageous that enough universes exist to take care of the problem.

There are probably as many models of multiverses as there are practitioners foolish enough to deal with the idea. In this section we shall play with a specific model, motivated by the idea of emergent field theories, a concept born from analogies with condensed matter physics [9]. The model will also be related to the ideas of evolu- 
tionary cosmology developed by Smolin [6], albeit in a more deterministic framework. As mentioned in the introduction, our reason for indulging in this fantasy is to try to obtain some guidance in the search for a satisfactory microscopic emergent theory.

The basic premise underlying the emergence approach is that the vacuum of particle physics and cosmology is analogous to a quantum liquid in equilibrium at very low temperature. Such a system has essentially zero pressure. But the measure of vacuum pressure is the cosmological constant itself, explaining not only why it should be zero, but why it is not quite zero: a droplet of vacuum of finite size will have pressure due to surface effects. This is just what happens in the DeSitter universe ( $c f$. Eq. (田) ).

Chapline et al. [8], and Mazur and Mottola [7, have recently carried this notion further, and argue that a black hole is to be considered a droplet of quantum liquid, with a nonsingular interior which is in fact static DeSitter space. The value of the cosmological constant in the interior differs from its value exterior to the horizon and serves as a kind of order parameter. In what we have described, this is generalized to all the standard model parameters, which evidently are also discontinuous across the horizon. This picture is ready-made for the cosmological setting in which we find ourselves: not only does our universe contain a large number of "daughter" black-hole fluid droplets, but our universe itself can be considered the interior of a much bigger droplet, which presumably exists, along with many other "sister" droplets, in a much larger "mother" universe. From this starting point, one easily sees that a genealogy can be defined. The properties of mother and daughter universes will depend upon how different in size they are from our own, and how differently the physics works at those size scales. It is this question that we take up in this section, building upon what was learned in the previous sections., We shall not venture very far beyond one generation in either direction; there will be more than enough uncertainty at this level.

It is easiest and most direct to first consider the daughter universes, because there 
are some data. There are at least two kinds of daughters - the supermassive, galactic black holes, of horizon size roughly 15 orders of magnitude smaller than the size of our universe, and the stellar-size black holes which are six to eight orders of magnitude smaller still. Despite the greater uncertainty in the underlying astrophysics, we specialize to the former because they are closest to us in size.

Rather than characterizing the black hole size by its horizon radius, it is also useful to give it in terms of the volume of comoving matter needed to form the black hole, which we assume is baryonic in origin. In our universe the mass of such black holes is in the range of $10^{6}$ to $10^{9}$ solar masses, or $10^{53}$ to $10^{56}$ proton masses, out of a total of about $10^{79}$ in the universe. So the fraction by volume of total comoving baryonic matter that ends up in one of these black holes is $10^{-23}$ to $10^{-26}$. Taking a cube root gives the fraction in linear scale of roughly $10^{-8}$ to $10^{-9}$. This should be compared with the fraction in linear scale of about $10^{-3}$ for the largest scale structures found in our universe.

Our main purpose in spinning out these numbers is to try to infer the most likely size of our mother universe. Evidently the first rough guess would be 15 powers of ten larger than our own. But by the time one goes out those fifteen orders of magnitude, the cosmology has significantly changed, and it is possible that one must go even further. Let us review what was learned in the previous section for the cosmology of a candidate mother universe, say, of radius $10^{45} \mathrm{~cm}$.

The early evolution of a mother universe of this size would be similar to our universe. Nucleosynthesis would occur in one of three possible scenarios (cf. Fig. 13), but in all cases hydrogen would predominate in the long run. The three cases are distinguished by the nonexistence of deuterium and/or the relative abundance of primordial helium. As the temperature decreased, decoupling of matter from radiation would occur during the radiation-dominated epoch (cf. Fig. 15). This means that the growth of density contrast would from the outset involve both the baryonic matter and the dark matter. However, the growth factor, which scales with the redshift at 
the time $t_{E Q}$ of matter-radiation equality, is less by about a factor ten than for our universe ( $c f$. Fig. 16). In addition, the ratio of baryonic to dark matter is much less; instead of ten percent, the number is somewhere between 3 percent and 0.01 percent. All of these features will make baryonic structure formation more difficult. What is most important for our consideration here is whether these mother universes can give birth to daughter black holes. Baryonic matter has to aggregate in the potential wells created by the dark matter and undergo gravitational collapse. While it apparently is more difficult for this to happen, it is not clear that the number of black holes that might be created is in fact small compared to unity, when for our universe the corresponding number (for "galactic" black holes) is $10^{10}$ or so. We shall make a guess that the fertility curve looks something like what is depicted in Fig. 17; this would allow mother universes to be present, but make it unlikely that grandmothers exist. But we must emphasize the many huge uncertainties involved, not the least of which is the assumption that the primordial fluctuation spectrum does not depend upon $R$.

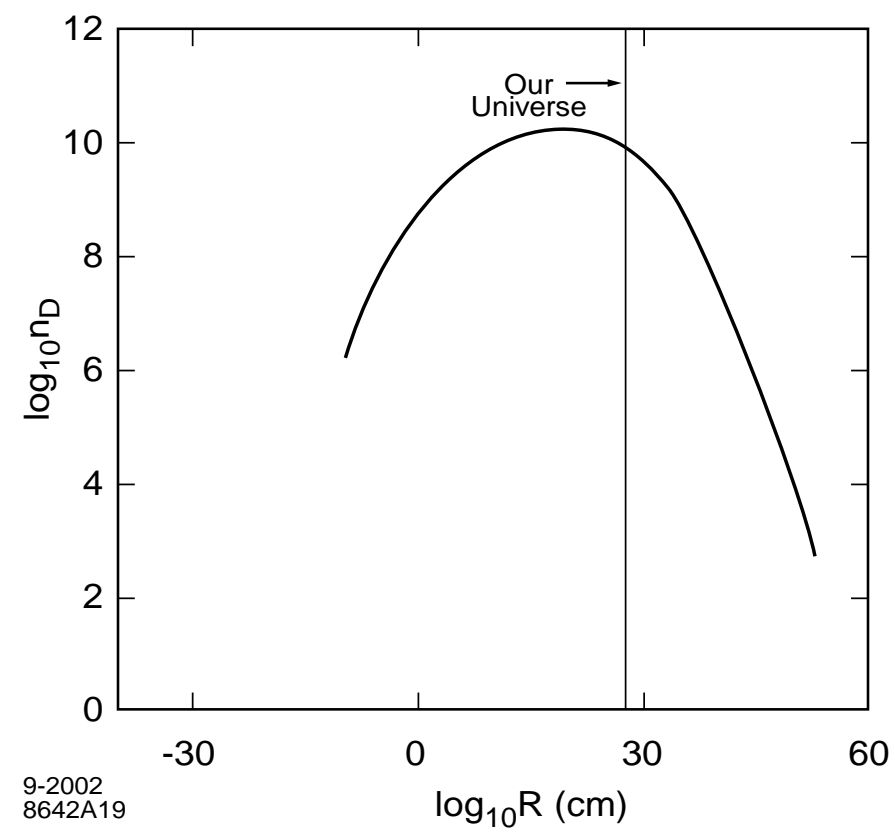

Figure 17: A guess for the dependence of fertility, i.e. the number $n_{D}$ of daughter universes per mother, upon size $R$. 
Relative to our universe, the fraction of matter in the mother universe which is baryonic is, as already mentioned, less than for our universe. This might affect not only the frequency of occurrence of black-hole formation, but also the size distribution. We do not try to estimate the effect, mainly out of lack of competence. But it is likely that the ratio of size of mother to daughter indeed grows with overall scale $R$, in the way sketched out in Fig. 18. But we emphasize that we are approaching a level of almost complete guesswork.

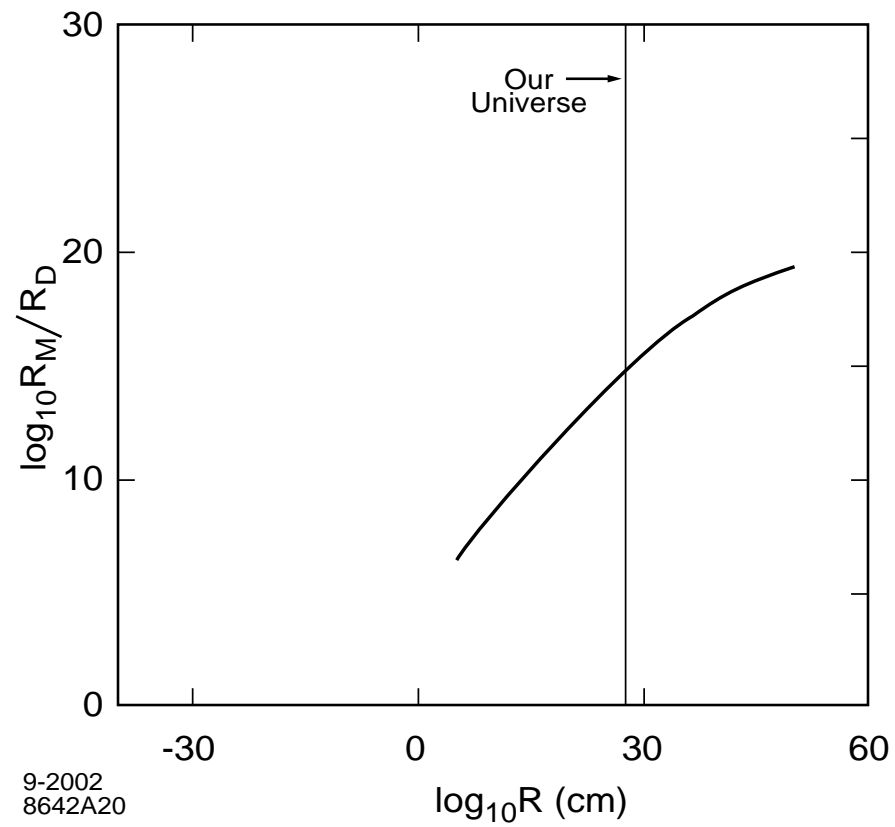

Figure 18: A guess for the ratio of the average size $R_{M}$ of a mother universe, assumed to be a supermassive black hole interior, to the size $R$ of its daughter, versus $R$.

Despite all these uncertainties, it seems relatively safe to conclude, given our assumptions, that the model of nested black holes for the multiverse allows at most one or two generations of parents, with a number of sister universes small compared to $10^{10}$, the number of (galactic) black hole daughters in our universe. It seems very unreasonable to assume a large number of "ancestor" generations, unless the primordial density fluctuations were to increase is magnitude with $R$. However, intuitively we would if anything expect the opposite to occur. 
Since the size distribution of sister universes span a few factors ten, the fraction with size close enough to our universe to in principle support life as we know it will be a few powers of ten less than the total population of sisters. This means that the total number of universes in such a nested-black-hole multiverse which could support life as we know it is bounded above by ten to a small power. It follows that the overall number of planets in the multiverse that are candidates for habitable environments is not all that different (on a logarithmic scale) from the number in our own universe.

The above inferences are rather strong, and therefore invite an additional critical look: are these conclusions avoidable? In such a soft topic as the contents of this paper, the answer is almost certainly yes. One assumption we have been making, mainly from a desire for simplicity and definiteness, is that the ensemble of universes we consider is parametrized only by the size $R$ and nothing else. The other cosmological parameters, such as magnitude of the baryon asymmetry and/or the magnitude of the primordial density fluctuations, may well represent independent initial conditions, unconstrained or at best loosely constrained by the value of the size parameter $R$. In such cases the conclusions about abundances of mother and grandmother universes are inoperative. Exploration of such alternatives seems however to be premature, and in any case beyond the scope of this paper.

\section{Lessons and Challenges}

While everything we have discussed is very speculative, it must be admitted that, given the starting hypothesis of size-dependent standard-model parameters, we have been able to look at old questions from a somewhat different perspective. This in itself can be a benefit, inasmuch as a fresh point of view is often a key to making

progress. And in fact there are some of the classic Big Questions for which partial answers can be set forward:

1. Why are there such hierarchies in scale amongst standard model parameters? 
This question includes the classic "hierarchy problem", namely the smallness of the electroweak scale $v$ relative to the Planck/GUT scale, which stimulates the introduction of weak-scale supersymmetry by so many practitioners. It also includes the question of why the electron mass is so much smaller than the top-quark mass. And it even includes the question of why the cosmological-constant scale is so much smaller than the QCD and electroweak scales, not to mention the Planck/GUT scale.

The answer to this general question, given the multiverse hypothesis, may be that for most universes in the ensemble there is no such huge hierarchy. If the size distribution of universes is maximum for relatively small values of $R$, say the GUT scale or smaller, then the typical universe has no large disparity of scales. Only the large, rare, universes like ours enjoy that property as a consequence of the assumed scaling behavior of parameters (which of course must eventually be explained).

2. Why is the fine-structure constant $1 / 137$ so small?

The answer to this famous old question is the same as above: in small universes $\alpha$ is not small; only in large ones like ours is it small. There are corollaries which are answered in the same way. The most immediate is the more modern version of the above question: why are the gauge coupling constants at the GUT unification scale so small? And directly related to this question is why the QCD scale $\Lambda_{Q C D}$ is so small relative to the GUT scale. All these questions are answered in the same way: because we live in a very large universe.

3. Why is our universe so large?

This is the obvious follow-up question to the previous ones. And the answer to this is weakly anthropic: our universe is large because we inhabit it. The discussions in the previous sections show it could not be otherwise, given the scaling assumptions underlying this note.

But in addition to these questions, there is the most important one, which remains without much of an answer:

4. Why should the assumed "fixed-point" scaling behavior be true? 
One response is that it (Fig. 3) looks just as credible as the conventional-wisdom alternative (Fig. 1) - which in itself sheds no light on the above questions. But at best this response is highly subjective and leaves much to be desired. To give a more satisfactory reply would be to relate the scaling behavior to the microscopic theory. This has not been done. But there are some interesting guidelines which the assumed behavior suggests. One concerns the limit of the standard model for infinite $R$. In that limit all dimensionless coupling constants vanish, and the standard model becomes trivial [2]. In other words, the presence of nontrivial interactions of the particles with each other depends upon the existence of a nonvanishing cosmological constant. In the emergent, "gravastar" scenario, this states that the standard-model interactions are present only because of the presence of the DeSitter horizon, in the neighborhood of which exists new, beyond-the-standard-model physics. It is as if all the standardmodel forces are in some sense Casimir effects. However, the standard kind of Casimir effect, which depends upon size of the system as an inverse power, will not do the trick. There are terms in the standard-model action, such as the Higgs mass term and the cosmological-constant term itself, which do have the typical behavior. But most of them, after appropriate rescaling of fields, depend only logarithmically upon the size parameter $R$. To see this, write schematically the standard model Lagrangian as

$$
\mathrm{E}=F^{2}+\bar{\psi} \not D \psi+(D \phi)^{2}+g \bar{\psi} \psi \phi+g^{2} \phi^{4}-\mu M \phi^{2}
$$

where the first three terms are gauge, fermion, and Higgs kinetic energy terms, and the last terms are Yukawa, quartic Higgs, and Higgs mass terms respectively.j The covariant derivative is

$$
D=\partial-g A
$$

and $g$ is a generic label for gauge or Higgs coupling; we take $\lambda \sim g^{2}$ because the

\footnotetext{
${ }^{\S}$ We here conjecture, as in our previous note [2], that the Higgs mass is the geometric mean of the cosmological and Planck/GUT scales.
} 
assumed $R$ dependence is then universal. Under the rescalings

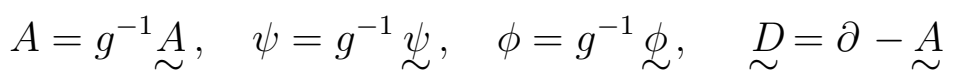

we find

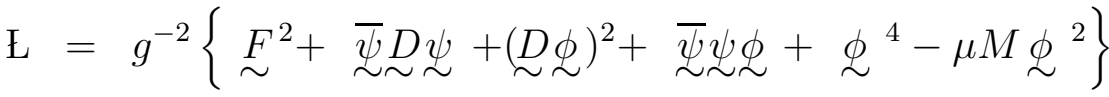

$$
\begin{aligned}
& \sim(\log M R) \stackrel{£}{\sim} .
\end{aligned}
$$

The action is

$$
S=\frac{1}{\hbar} \int d^{4} x \mathrm{E}=\frac{1}{\hbar(R)} \int d^{4} x \stackrel{\mathrm{E}}{\sim}
$$

with

$$
\hbar(R) \sim \frac{1}{(\ln M R)}
$$

The entire Lagrangian density gets multiplied by a factor $\ell n M_{p l} R$, as if the Planck constant itself is scale-dependent, vanishing in the limit of infinite $R$.

We have not considered in this note such a possibility, and have in fact essentially set the Planck constant, the speed of light, and the Planck mass to unity, not allowing them to vary with $R$. As long as the universes in the multiverse are causally disconnected from each other, this can be defended as no more than a convention in the choice of units [24]. However, if there is a connection between the universes, such as in the nested-black-hole scenario, then it is no longer obvious that this is a safe assumption. Relaxation of such an assumption might in fact lead to additional insight. However, exploration of this possibility is beyond the scope of this work.

In addition to the questions above, for which our approach might provide some insight, there are others for which our present lack of understanding is highlighted, and which need better answers in order to sharpen the consequences of the scaling assumptions which we have made. These include:

5. What is the mechanism by which the electron and the light quarks get their mass?

This is often viewed as a minor detail in the grand scheme of standard model problems. But in the context of this note, the lack of understanding of the origin of 
light quark and lepton masses is translated into relatively great uncertainty in the understanding of the relationship of our universe to other universes of different size.

6. What is the nature of the dark matter?

7. What is the origin of the baryon asymmetry, and what determines its magnitude of $\eta=3 \times 10^{-10}$ ?

8. Why is the value of the primordial density fluctuations $(\delta \rho / \rho)_{0}$ equal to $2 \times 10^{-5}$ ?

These three cosmological questions are hardly novel [25]; they are evidently crucial to better understanding the properties of the ensemble of universes we consider. Perhaps the only novelty is that we omit (here) the question of the "small" cosmological constant, usually added to the above list.

It is also worth noting that, while the flatness problem (why $\Omega=1$ ) and the scale-invariant spectrum of primordial fluctuations represent something of a triumph for the idea of inflation, there remains no good answer to the eighth question: the magnitude of the primordial fluctuation spectrum is simply fit to the data, and not understood at all from more fundamental considerations. And, as discussed at the end of the previous section, it is possible that these parameters should be considered as independent characterizations of members of the ensemble of universes, i.e. as initial conditions not strongly dependent upon the size parameter $R$.

Finally, there are the lessons, if any, which are learned from this exercise that may be applied to the hypothesis of emergence. The idea of emergence provides some motivation for the scaling behavior assumed from the beginning of this note. But it has many daunting problems associated with it:

1. Why are violations of Lorentz covariance so small?

Condensed-matter analogs of emergence suggest in general that symmetries such as Lorentz covariance are just low energy approximations. At high enough energies deviations are to be expected. But experiment severely limits such deviations. For example, noncovariant corrections to charge-renormalization, an ultraviolet-sensitive quantity, are limited [26] to less than one part in $10^{31}$. This comprises a staggeringly 
restrictive constraint. It would seem essential that there be a very small parameter which characterizes the violations. And the scaling behavior of parameters studied here suggests that a necessary (but far from sufficient) condition for the Lorentzviolating terms in the Lagrangian is that they scale as inverse powers of the radius $R$ of the universe. If this is so, then very small universes exhibit very little symmetry, while the very large ones like our own exhibit Lorentz symmetry, etc., with very small corrections.

\section{What is the structure of event horizons?}

In the nested-black-hole, or "gravastar" scenario, there is "new physics" at horizons. This is endemic in the condensed-matter analogues [10. And in our picture, standard-model parameters (including the cosmological constant) are discontinuous across horizons, indicating that at the surface of discontinuity conventional-physics descriptions of what is going on are incomplete. There also appear to be violations of the weak energy conditions of classical general relativity [27]. One manifestation of this appears to be that there are large classes of null geodesics (in particular those which have nontrivial transverse motion) which are "bound" to the horizon. There is a nontrivial problem here of providing a consistent description.

In addition, if our universe is to be regarded as the DeSitter interior of a gravastar, then there must be in our universe preferred comoving observers, presumably not ourselves, with respect to which there is the "physical" horizon associated with our black-hole interior. It then becomes an interesting question as to where we should regard ourselves relative to these central observers: how far away are they, and in what direction? Might there be observational issues associated with this preferred center of our universe? While these are quite interesting questions, they also lie beyond the scope of this note.

\section{How are gravastars formed?}

If the gravastar picture is in fact viable, then there must be a time evolution of the "new physics" which is associated with the horizon. But for large black holes, it is hard 
to find an intrinsic, local parameter associated with the horizon, because classically it can be regarded as an artifact associated with a choice of coordinate systems. In the emergence scenario, general covariance is only a low energy approximation. This implies that the description of gravastar formation will require a "best" choice of coordinates. What should be chosen?

This is only one of the difficult issues involving gravastar formation. Another involves rotation: no "eternal rotating gravastar" generalization of the nonrotating case has been found. In nested-black hole cosmologies, it is necessary that all the black-hole universes (including ours) are characterized by a value of spin as well as mass. This is not only a complication, but also an opportunity for linking standard model discrete symmetry violations, in particular $C P$, to the existence of a spin axis for the new physics at the DeSitter horizon - physics which presumably controls the nontrivial interaction features of the standard model.

On the more positive side, some insight on the history of gravastar formation might be gleaned by comparing the formation of a daughter black hole with the formation of our own parent universe. The characteristic time for the formation of the daughter can be easily taken to be at the very least many millions of years, a timescale much larger than the size of the gravastar. If we assume the same for our parent universe, it follows that the formation time for our universe should be considered to be much larger than the size parameter $R$-in other words orders of magnitude larger than $10^{10}$ years [28]. This might imply that the formation time of the "new physics" on the DeSitter horizon likewise is long compared to $10^{10}$ years. Some kind of cosmological "bounce" scenario [29] might have the best chance of providing a concrete implementation of this inference.

\section{What is the microscopic physics underlying the emergence scenario?}

This question remains unanswered. Necessary conditions are that the gauge

"A default option is to invoke an "eye of the hurricane" model. Choose the Kerr metric for the exterior, and static DeSitter space for the interior. Then build an appropriate interpolating boundary layer with an exotic spacetime, which probably contains vorticity. 
bosons of the standard model, as well as the graviton, should be considered collective modes of the presumed "quantum liquid" vacuum. They quite likely should be all considered Goldstone modes [2, 30] associated with various kinds of spontaneous symmetry breakdown. The pattern of internal symmetries, especially in the fermion representations, must be an essential clue.

Finding the answer to this last question may well afford the best chance of turning the very speculative material in this note into something considerably more concrete.

\section{Acknowledgments}

It is a pleasure to thank my colleagues at Stanford for many helpful discussions, especially R. Adler, P. Chen, and M. Weinstein. I also thank S. Beane and M. Savage for valuable discussions on the chiral limit of nuclear physics.

\section{References}

[1] For reviews, see e.g. S. Carroll, astro-ph/0004075; astro-ph/0107571; A. Liddle, hep-ph/0201064.

[2] J. Bjorken, Phys. Rev. D64, 85008 (2001), hep-ph/0103349; hep-th/0111196.

[3] M. Rees, "Our Cosmic Habitat" (Princeton University Press, Princeton NJ, 2001).

[4] J. Barrow and F. Tipler, "The Anthropic Cosmological Principle" (Oxford University Press, Oxford, 1986).

[5] D. Brownlee and P. Ward, "Rare Earth" (Copernicus, Springer-Verlag, New York NY, 1999).

[6] L. Smolin, gr-qc/9404011; "The Life of the Cosmos" (Crown Press, New York NY, 1995). 
[7] P. Mazur and E. Mottola, gr-qc/0109035.

[8] G. Chapline, E. Hohlfeld, R. Laughlin, and D. Santiago, gr-qc/0012094.

[9] G. Volovik, Phys. Repts. 351, 58 (201), gr-qc/000509; "The Universe in a Helium Droplet" (Oxford University Press, to be published).

[10] C. Barcelo, S. Liberati, and M. Visser, gr-qc/010400; gr-qc/0106002, and references therein.

[11] J. Gasser and H. Leutwyler, Phys. Rept. 87, 77 (1982).

[12] B. Carter and M. Rees, Nature 278, 605 (1979).

[13] V. Flambaum and E. Shuryak, hep-ph/0201303

[14] M. Fairbairn, hep-ph/0205078.

[15] S. Beane and M. Savage, hep-ph/0206113; nucl-th/0208021.

[16] E. Epelbaum, U.-G. Meissner, and W. Glockle, nucl-th/027089.

[17] For a nice historial review, cf. R. Spear, The Physicist 39, 35 (2000).

[18] F. Hoyle, Ap. J. Suppl. 1, 121 (1954); F. Hoyle, D. Dunbar, W. Wenzel, and W. Whaling, Phys. Rev. 92, 1095 (1953).

[19] H. Oberhummer, R. Pichler, and A. Csoto, nucl-th/9810057.

[20] M. Livio, D. Hollowell, A. Weiss, and J. Truran, Nature 340, 281 (1989).

[21] E. Kolb and M. Turner, "The Early Universe" (Perseus, Cambridge MA, 1990).

[22] See e.g. G. Jungman, M. Kamionkowski, and K. Greist, Phys. Rept. 267, 195 (1996).

[23] We identify closely with the interpretation of the anthropic principle expressed by B. Muller, astro-ph/0108259. 
[24] M. Duff, hep-th/0208093; M. Duff, L. Okun, and G. Veneziano, JHEP0203, 023 (2002), physics/0110060.

[25] See for example M. Rees, "Just Six Numbers"; Basic Books, New York NY (2000).

[26] V. Kostelecky and M. Mewes, hep-ph/0111026.

[27] See for example E. Farhi and A. Guth, Phys. Lett. B183, 149 (1987); E. Poisson and W. Israel, Class. Quantum Grav. 5, L201 (1988).

[28] We somewhat share the opinion of M. Dyson, L. Kleban, and L. Susskind, hepth/0208013, that "dismissing such long times as 'unphysical' may be a symptom of extreme temporal provincialism".

[29] See for example A. Eason and R. Brandenberger, hep-th/0103019; I. Dymnikova, A. Dobosz, M. Filchenkov, and A. Gromov, gr-qc/0102032, and references therein.

[30] P. Kraus and E. Tomboulis, hep-th/0203221. 